\title{
Closed-Loop Supply Chain Network under Oligopolistic Competition with Multiproducts, Uncertain Demands, and Returns
}

\author{
Yan Zhou, ${ }^{1}$ Chi Kin Chan, ${ }^{2}$ Kar Hung Wong, ${ }^{3}$ and Y. C. E. Lee ${ }^{2}$ \\ ${ }^{1}$ Department of Management Science and Engineering, Qingdao University, Qingdao, China \\ ${ }^{2}$ Department of Applied Mathematics, The Hong Kong Polytechnic University, Kowloon, Hong Kong \\ ${ }^{3}$ School of Computational and Applied Mathematics, University of the Witwatersrand, Johannesburg, South Africa
}

Correspondence should be addressed to Yan Zhou; yanyanz22@hotmail.com

Received 29 January 2014; Revised 20 April 2014; Accepted 27 April 2014; Published 27 May 2014

Academic Editor: Changzhi Wu

Copyright (C) 2014 Yan Zhou et al. This is an open access article distributed under the Creative Commons Attribution License, which permits unrestricted use, distribution, and reproduction in any medium, provided the original work is properly cited.

\begin{abstract}
We develop an equilibrium model of a closed-loop supply chain (CLSC) network with multiproducts, uncertain demands, and returns. This model belongs to the context of oligopolistic firms that compete noncooperatively in a Cournot-Nash framework under a stochastic environment. To satisfy the demands, we use two different channels: manufacturing new products and remanufacturing returned products through recycling used components. Since both the demands and product returns are uncertain, we consider two types of risks: overstocking and understocking of multiproducts in the forward supply chain. Then we set up the Cournot-Nash equilibrium conditions of the CLSC network whereby we maximize every oligopolistic firm's expected profit by deciding the production quantities of each new product as well as the path flows of each product on the forward supply chain. Furthermore, we formulate the Cournot-Nash equilibrium conditions of the CLSC network as a variational inequality and prove the existence and the monotonicity of the variational inequality. Finally, numerical examples are presented to illustrate the efficiency of our model.
\end{abstract}

\section{Introduction}

The competitive supply chain network models have been widely studied in the past decade. For instance, Nagurney et al. [1] first considered an equilibrium model of a competitive forward supply chain network which consisted of a lot of decision makers. Dong et al. [2] constructed an equilibrium model of a forward supply chain network under the assumption that the demands were uncertain. Nagurney and Toyasaki [3] constructed an equilibrium model of a reverse supply chain network for the optimal management of the electronic wastes. Hammond and Beullens [4] expanded the work of $[1,3]$ by presenting some insights of how closedloop supply chain (CLSC) network equilibrium was achieved under WEEE (the waste electrical and the waste electrical and electronic equipment) directive legislation. Yang et al. [5] constructed an equilibrium model of a CLSC network consisting of raw materials, suppliers, and recovery centers besides manufacturers and retailers. In all the above papers
[1-5], the equilibrium conditions were obtained by the theory of variational inequality; the variational inequalities in these papers were solved by the modified projection method proposed by Korpelevich [6].

The CLSC integrating the forward and reverse supply chain is important in real world applications due to government legislations (such as the paper recycling directive and WEEE within the European Union [7]). Moreover, the process used in the CLSC to recycle used products (such as papers, glass, building wastes, electric, and electronic equipment) for minimizing resource wastage also leads to people's understanding of the important concept of the green supply chain management (GSCM). A comprehensive review of the CLSC management and the GSCM can be found in Gupta and Palsule-Desai [8], Sheu and Talley [9], and Seuring [10].

Competition and monopoly are two opposing market forms in economics. In a competition, there are numerous firms supplying their goods to the markets and the market price is determined exogenously. In a monopoly, only one 
firm supplies its goods to the markets. Oligopoly is a market form between perfect competition and monopoly, in which the market is dominated by $n$ firms (oligopolists), where $n$ is a small number. The $n$ firms compete in the oligopolistic market by producing the same product or perfect substitutes for the maximization of their profits, considering the best reply of the other firms. (When $n=2$, the oligopoly is called a duopoly which was first proposed by Cournot [11].) Recently, Barbagallo and Cojocaru [12] considered the dynamic equilibrium of an oligopolistic market. Based on the timedependent variational inequality, Barbagallo and Mauro [13] investigated an equilibrium model of an oligopolistic market dealing with production and demand excesses.

From the work of Brown et al. [14], it is clear that more firms nowadays are aware of the importance of integrating the supply chain as a whole, consisting of all the marketing activities of all the competitors. Thus, several forward supply chain networks involving oligopolistic competition among firms have been developed. For instance, Nagurney [15] considered a design problem in which oligopolistic firms competed in a Cournot-Nash $[16,17]$ framework. Masoumi et al. [18], Nagurney and Yu [19], and Yu and Nagurney [20] developed equilibrium models involving oligopolistic competition among pharmaceutical firms, fashion firms, and fresh food firms, respectively. The equilibrium conditions of [15, 18-20] were also obtained by the theory of variational inequality; the variational inequalities in these papers were solved by the Euler method [21]. However, there are very few papers in the literature dealing with CLSC network involving oligopolistic competition among firms and none of these papers consider oligopolistic competition in a stochastic environment.

The major weakness of the literature concerning the CLSC network model is that only deterministic demands are considered. Research work on stochastic CLSC network model began only in the last few years. For instance, Qiang et al. [22] investigated an equilibrium model of a CLSC network involving perfect competition and uncertainties in demands, but not involving uncertainties in returns. Shi et al. $[23,24]$ constructed mathematical models of closedloop manufacturing systems, in which both the demands and the returns were uncertain and price sensitive. However, the closed-loop systems in $[23,24]$ were not CLSC networks and did not involve oligopolistic competition among firms.

In this paper, we provide an innovative framework to study the effects of oligopolistic competition on a CLSC network and construct an equilibrium model of a multiproduct CLSC network involving oligopolistic competition among firms, by allowing uncertain demands and returns for all products. To satisfy these demands, we use two different channels: manufacturing new products and remanufacturing returned products through recycling used components. Since the demands and returns of all products are uncertain, we consider two types of risks: overstocking and understocking of multiproducts in the forward supply chain. Then we can set up the Cournot-Nash equilibrium conditions of the CLSC network whereby we maximize every oligopolistic firm's expected profit by deciding the production quantities of each new product as well as the path flows of each product on

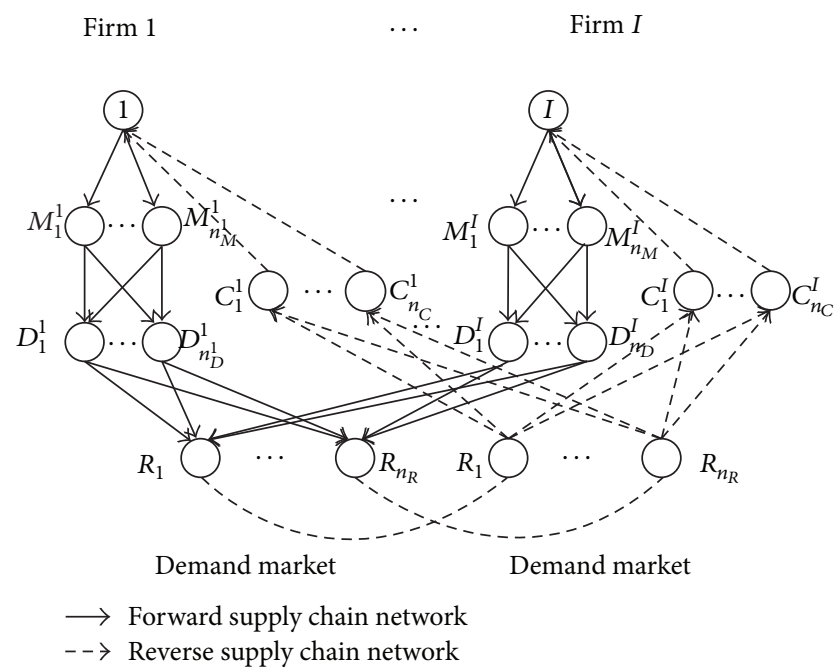

FIGURE 1: The CLSC network topology.

the forward supply chain. Since the CLSC network model constructed in the above manner takes into consideration all those important issues such as competition among oligopolistic firms with multiproduct as well as uncertainty in both demands and returns, the CLSC model developed in this paper has more practical use than most of them in the literature, such as those developed in $[4,5,22]$.

The rest of this paper is organized as follows. Section 2 develops the oligopolistic CLSC network model with multiproducts and uncertain demands and returns and constructs the Cournot-Nash equilibrium conditions. Section 3 gives the variational inequality formulations of our model. We then prove the existence and the monotonicity results for our model in Section 4. Section 5 presents an algorithm for finding the Cournot-Nash equilibrium. In Section 6, numerical examples are presented to test the effectiveness of the proposed algorithm. Sensitivity analysis is also conducted to illustrate how the change in a parameter can affect the firms' strategies and their respective expected profits. Section 7 presents our summary and conclusion.

\section{The Multiproduct CLSC Network Model under Oligopolistic Competition among Firms}

We consider a CLSC network consisting of $I$ oligopolistic firms who compete noncooperatively. Each firm $i(i=1$, $\ldots, I)$ manufactures $J$ products and has two different channels to meet the demands: manufacturing new products and remanufacturing returned products through recycling used components. Both the demands and their returns for all products are uncertain. It is assumed that all the demands and all the returns for each product are random variables with known probability distributions. The problem is to maximize all the oligopolistic firms' expected profits by deciding the production quantities of each new product as well as the path flows of each product on the forward supply chain.

The topology of this network is shown in Figure 1. From Figure 1, the CLSC network consists of the forward supply 
chain network and the reverse supply chain network. Each link in Figure 1 represents an activity of the CLSC network. Consider any firm $i(i=1, \ldots, I)$ in the CLSC network. The economic activities in the forward supply chain network can be described as follows.

(1) Firm $i$ distributes raw materials or recycled products to each of its manufacturers (denoted by $M_{1}^{i}$, $\left.\ldots, M_{n_{M}^{i}}^{i}\right)$.

(2) The manufacturers then produce new products or remanufactured products and distribute them to each of the distribution centers of firm $i$ (denoted by $D_{1}^{i}$, $\left.\ldots, D_{n_{D}^{i}}^{i}\right)$.

(3) The distribution centers then distribute these products to each of the demand markets (denoted by $\left.R_{1}, \ldots, R_{n_{R}}\right)$.

The economic activities of firm $i$ in the reverse supply chain network can be described as follows.

(1) The demand markets send a fraction of the used products to each of the recovery centers of firm $i$ (denoted by $C_{1}^{i}, \ldots, C_{n_{C}^{i}}^{i}$ ). (The remainder of the used products will be sent to the landfill for disposition.)

(2) The recovery centers then recycle the used products and send the recycled products to firm $i$ for remanufacturing.

Thus, our CLSC network is one which incorporates the forward supply chain processes (such as production, remanufacturing, delivery, and distribution) with the reverse supply chain processes (such as disposal, recycling, and returning) to form a loop in Figure 1.

We first give a few basic assumptions, which are common assumptions in the CLSC literature (see $[4,23]$ for details).

Assumption A. (A1) The demand for product $j(j=1, \ldots, J)$ of firm $i(i=1, \ldots, I)$ at demand market $R_{k}\left(k=1, \ldots, n_{R}\right)$, denoted by $\widehat{d}_{i j k}$, is a random variable. Any two demands are independent of each other.

(A2) The return of product $j(j=1, \ldots, J)$ from demand market $R_{k}\left(k=1, \ldots, n_{R}\right)$ to firm $i(i=1, \ldots, I)$, denoted by $\widehat{r}_{k j i}$, is a random variable. Any two returns are independent of each other.

(A3) The qualities of new products and remanufactured products are exactly the same.

(A4) A fraction of the used products will be collected by the firms for remanufacturing; the uncollected used products will be sent from the market centers to the landfill for disposition. The firm will incur a fee of $\bar{\rho}$ per unit for the disposal of these used products at the landfill site.

(A5) For the collected products mentioned in (A4), the firm will incur a cost of $\rho_{j}^{\mathrm{Re}}$ per item for the purchase of the returned product $j(j=1, \ldots, J)$. It is also assumed that all returns are remanufacturable.

(A6) The quantity of new product $j(j=1, \ldots, J)$ manufactured by firm $i(i=1, \ldots, I)$ cannot exceed $\bar{x}$, where $\bar{x}$ is a positive integer.
In order to formulate the problem of finding the CournotNash equilibrium for our CLSC network, we need to first describe the decision variables.

For each $i(i=1, \ldots, I)$ and $j(j=1, \ldots, J)$, let $x_{i j}^{\text {New }}$ denote the quantity of new product $j$ manufactured by firm $i$.

In the forward supply chain, any set of correlated links connecting the firms to the demand markets via the manufacturers and the distribution centers form a path $p^{f}$. For each $i$ $(i=1, \ldots, I)$ and $k\left(k=1, \ldots, n_{R}\right)$, let $P_{k}^{i}$ denote the set of all the forward paths from firm $i$ to demand market $R_{k}$. Then for each $p^{f} \in P_{k}^{i}$, let $x_{j p^{f}}$ denote the nonnegative product flow of product $j(j=1, \ldots, J)$ on the forward path $p^{f}$.

Hence there are two types of decision variables for our problem, namely, the quantity of new product $x_{i j}^{\mathrm{New}}$ and the product flow on the forward path $x_{j p^{f}}$.

Let

$$
X_{i j} \equiv\left\{\left(x_{j p^{f}}, x_{i j}^{\mathrm{New}}\right) \mid p^{f} \in P^{i}\right\} \in \mathbb{R}_{+}^{n_{p^{i}}+1}
$$

denote all the decision variables associated with firm $i$ and product $j$, where

$$
P^{i} \equiv \bigcup_{k=1}^{n_{R}} P_{k}^{i}
$$

and $n_{P^{i}}$ denotes the cardinality of $P^{i}$.

Let

$$
X_{i} \equiv\left(X_{i 1}, \ldots, X_{i J}\right)^{T} \in K_{i}
$$

be the strategy vector representing the overall decision variables associated with firm $i$, where $K_{i} \equiv \mathbb{R}_{+}^{\left(n_{P^{i}}+1\right) J}$. Then

$$
X \equiv\left(X_{11}, \ldots, X_{1 J}, \ldots, X_{i 1}, \ldots, X_{i J}, \ldots, X_{I 1}, \ldots, X_{I J}\right)^{T} \in K
$$

is the overall decision variables for the entire CLSC network, which is the strategies vector of all firms, where $K \equiv$ $\mathbb{R}_{+}^{\sum_{i=1}^{I}\left(n_{p^{i}}+1\right) J}$.

Now, we consider all the different types of constraints for our CLSC network.

By virtue of (A6), we have for each $i(i=1, \ldots, I)$ and $j$ $(j=1, \ldots, J)$

$$
x_{i j}^{\mathrm{New}} \leq \bar{x}
$$

Moreover, in the forward supply chain, the following inequality involving product $j(j=1, \ldots, J)$ manufactured by firm $i(i=1, \ldots, I)$ must hold:

$$
\sum_{k=1}^{n_{R}} \sum_{p^{f} \in P_{k}^{i}} x_{j p^{f}} \leq x_{i j}^{\mathrm{New}}+E\left(x_{i j}^{\mathrm{Re}}\right),
$$

where

$$
x_{i j}^{\mathrm{Re}}=\sum_{k=1}^{n_{R}} \widehat{r}_{k j i}
$$

and $\widehat{r}_{k j i}$ is as defined in (A2). 
Furthermore, in the reverse supply chain, the following flow inequality must hold:

$$
E\left(\widehat{r}_{k j i}\right) \leq \sum_{p^{f} \in P_{k}^{i}} x_{j p^{f}} .
$$

The above inequality ensures that the expected value of the returned product $j(j=1, \ldots, J)$ from demand market $R_{k}$ $\left(k=1, \ldots, n_{R}\right)$ to firm $i(i=1, \ldots, I)$ cannot exceed the total amount of forward flows of the above product on any path connecting firm $i$ to demand market $R_{k}$.

Now, we formulate the different cost functions for our CLSC network. We first formulate the expected penalty cost (due to excessive or insufficient supply) and the operational cost.

Consider the forward supply chain involving firm $i(i=$ $1, \ldots, I)$, product $j(j=1, \ldots, J)$, and demand market $R_{k}$ $\left(k=1, \ldots, n_{R}\right)$. Let $v_{i j k}$ denote the quantity of product $j$ supplied by firm $i$ to demand market $R_{k}$. Then the following flow equation must hold:

$$
\sum_{p^{f} \in P_{k}^{i}} x_{j p^{f}}=v_{i j k}
$$

Let $\widehat{d}_{i j k}$ denote the demand associated with firm $i$, product $j$, and demand market $R_{k}$. Let $\mathscr{F}_{i j k}$ be the probability density function of $\widehat{d}_{i j k}$. Then

$$
\operatorname{Pr}\left(\widehat{d}_{i j k} \leq x\right)=\int_{0}^{x} \mathscr{F}_{i j k}\left(\widehat{d}_{i j k}\right) \mathrm{d} \widehat{d}_{i j k},
$$

where Pr denotes the probability.

Now, the quantity of product $j$ supplied by firm $i$ to demand market $R_{k}$ cannot exceed the minimum of $v_{i j k}$ and $\widehat{d}_{i j k}$. In other words, the actual sale of these products is equal to $\min \left\{v_{i j k}, \widehat{d}_{i j k}\right\}$.

Let

$$
\begin{aligned}
& \Delta_{i j k}^{+}=\max \left\{0, v_{i j k}-\widehat{d}_{i j k}\right\}, \\
& \Delta_{i j k}^{-}=\max \left\{0, \widehat{d}_{i j k}-v_{i j k}\right\},
\end{aligned}
$$

denote, respectively, the quantity of the overstocking and the understocking of goods associated with firm $i$, product $j$ and demand market $R_{k}$.

The expected values of $\Delta_{i j k}^{+}$, and $\Delta_{i j k}^{-}$are given by

$$
\begin{aligned}
& E\left(\Delta_{i j k}^{+}\right)=\int_{0}^{v_{i j k}}\left(v_{i j k}-\widehat{d}_{i j k}\right) \mathscr{F}_{i j k}\left(\widehat{d}_{i j k}\right) \mathrm{d} \widehat{d}_{i j k}, \\
& E\left(\Delta_{i j k}^{-}\right)=\int_{v_{i j k}}^{+\infty}\left(\widehat{d}_{i j k}-v_{i j k}\right) \mathscr{F}_{i j k}\left(\widehat{d}_{i j k}\right) \mathrm{d} \widehat{d}_{i j k} .
\end{aligned}
$$

Assume that the unit penalty incurred on firm $i$ due to excessive supply of product $j$ to demand market $R_{k}$ is $\theta_{i j k}^{+}$and the unit penalty incurred on firm $i$ due to insufficient supply of product $j$ to demand market $R_{k}$ is $\theta_{i j k}^{-}$, where $\theta_{i j k}^{+} \geq 0$ and $\theta_{i j k}^{-} \geq 0$. Then, the total expected penalty cost incurred on firm $i$ associated with product $j$ and demand market $R_{k}$ is given by

$$
E\left(\theta_{i j k}^{+} \Delta_{i j k}^{+}+\theta_{i j k}^{-} \Delta_{i j k}^{-}\right)=\theta_{i j k}^{+} E\left(\Delta_{i j k}^{+}\right)+\theta_{i j k}^{-} E\left(\Delta_{i j k}^{-}\right) .
$$

Let $L^{f}$ be the set of all correlated links in the forward supply chain. Let $u_{j a}^{f}$ denote the product flow of product $j$ on any link $a$ in the forward supply chain. Then, in the forward supply chain involving product $j$, the relationship between the product flow in a link and the product flow in a path is as follows:

$$
u_{j a}^{f}=\sum_{i=1}^{I} \sum_{k=1}^{n_{R}} \sum_{p^{f} \in P_{k}^{i}} x_{j p^{f}} \delta_{a p^{f}}, \quad \forall a \in L^{f},
$$

where the binary parameter $\delta_{a p^{f}}$ is used to indicate whether link $a$ is included in forward path $p^{f}\left(\delta_{a p}=1\right)$ or not included in forward path $p^{f}\left(\delta_{a p^{f}}=0\right)$. Let $u_{j}^{f}$ be the vector consisting of all the forward link flows for each product $j$.

Then, in the forward supply chain, the total operational cost of product $j$ on a link $a$ is a continuous function of all the forward link flows of product $j$ given by

$$
\widehat{c}_{j a}=\widehat{c}_{j a}\left(u_{j}^{f}\right), \quad \forall a \in L^{f} .
$$

Apart from the penalty costs given by (13) (due to excessive or insufficient supply) and the operational costs given by (15), firm $i(i=1, \ldots, I)$ also incurs the following additional costs:

(i) the cost related to the production of new product $j$ $(j=1, \ldots, J)$ :

$$
\bar{f}_{i j}\left(x_{i j}^{\mathrm{New}}\right) \text {, }
$$

where $\bar{f}_{i j}$ is a continuous function,

(ii) the cost related to the production of the returned product $j(j=1, \ldots, J)$ :

$$
\bar{f}_{i j}^{\mathrm{Re}}\left(x_{i j}^{\mathrm{Re}}\right) \text {, }
$$

where $\bar{f}_{i j}^{\mathrm{Re}}$ is a continuous function,

(iii) the cost related to the purchase of returned product $j$ $(j=1, \ldots, J)$ from demand market $R_{k}\left(k=1, \ldots, n_{R}\right)$ to firm $i$ :

$$
\rho_{j}^{\mathrm{Re}} \widehat{r}_{k j i},
$$

where $\rho_{j}^{\mathrm{Re}}$ is the purchase cost per item of these returned products,

(iv) the shipping cost related to the transportation of returned product $j(j=1, \ldots, J)$ from demand market $R_{k}\left(k=1, \ldots, n_{R}\right)$ to firm $i$ :

$$
\widehat{c}_{k j i}^{\mathrm{Re}} \widehat{r}_{k j i} \text {, }
$$

where $\hat{c}_{k j i}^{\mathrm{Re}}$ is the transportation cost per item of these returned products, 
(v) the cost related to the disposal of uncollected product associated with firm $i$, product $j(j=1, \ldots, J)$, and demand market $R_{k}\left(k=1, \ldots, n_{R}\right)$ at the landfill site:

$$
\bar{\rho}\left(\sum_{p^{f} \in P_{k}^{i}} x_{j p^{f}}-\widehat{r}_{k j i}\right) .
$$

By virtue of (13), (15), and (16)-(20), the total cost incurred on firm $i$ is given by

$$
\begin{aligned}
& \sum_{j=1}^{J} \sum_{k=1}^{n_{R}} E\left(\theta_{i j k}^{+} \Delta_{i j k}^{+}+\theta_{i j k}^{-} \Delta_{i j k}^{-}\right) \\
& \quad+\sum_{j=1}^{J} \bar{f}_{i j}\left(x_{i j}^{\mathrm{New}}\right)+\sum_{j=1}^{J} \sum_{a \in L_{i}^{f}} \widehat{c}_{j a}\left(u_{j}^{f}\right) \\
& +\sum_{j=1}^{J} \sum_{k=1}^{n_{R}} \rho_{j}^{\mathrm{Re}} E\left(\widehat{r}_{k j i}\right)+\sum_{j=1}^{J} E\left(\bar{f}_{i j}^{\mathrm{Re}}\left(x_{i j}^{\mathrm{Re}}\right)\right) \\
& \quad+\sum_{j=1}^{J} \sum_{k=1}^{n_{R}} \widehat{c}_{k j i}^{\mathrm{Re}} E\left(\widehat{r}_{k j i}\right)+\bar{\rho} \sum_{j=1}^{J} \sum_{k=1}^{n_{R}} E\left(\sum_{p^{f} \in P_{k}^{i}} x_{j p^{f}}-\widehat{r}_{k j i}\right),
\end{aligned}
$$

where $L_{i}^{f}$ is the set of all correlated links in the forward supply chain of firm $i(i=1, \ldots, I)$.

Now, we formulate the total revenue received by firm $i$ ( $i=$ $1, \ldots, I)$. In order to capture competition in the demands in the entire CLSC network, we assumed that, for each product $j$, the demand price function $\rho_{i j k}\left(j=1, \ldots, J, k=1, \ldots, n_{R}\right)$ is a continuous function of all the demands of product $j$ in the entire CLSC network; that is,

$$
\rho_{i j k}=\rho_{i j k}\left(\widehat{d}_{j}\right)
$$

where

$$
\widehat{d}_{j}=\left(\widehat{d}_{1 j 1}, \ldots, \widehat{d}_{1 j n_{R}}, \ldots, \widehat{d}_{I j 1}, \ldots, \widehat{d}_{I j n_{R}}\right)^{T} \in \mathbb{R}_{+}^{I \times n_{R}}
$$

denotes the vector consisting of all the demands of product $j$ in the entire CLSC network. Masoumi et al. [18] and Nagurney and $\mathrm{Yu}$ [19] used such demand price function in the study of forward supply chain network involving oligopolistic competition among firms. Then the expected revenue received by firm $i(i=1, \ldots, I)$ is given by

$$
\sum_{j=1}^{J} \sum_{k=1}^{n_{R}} E\left(\rho_{i j k}\left(\widehat{d}_{j}\right) \min \left\{v_{i j k}, \widehat{d}_{i j k}\right\}\right) .
$$

By virtue of the revenue, the cost of the forward supply chain, and the cost of the reverse supply chain, the expected profit function of firm $i(i=1, \ldots, I)$, denoted by $U_{i}$, can be expressed as follows:

$$
\begin{aligned}
U_{i}= & \sum_{j=1}^{J} \sum_{k=1}^{n_{R}} E\left(\rho_{i j k}\left(\widehat{d}_{j}\right) \min \left\{v_{i j k}, \widehat{d}_{i j k}\right\}\right) \\
& -\sum_{j=1}^{J} \sum_{k=1}^{n_{R}} E\left(\theta_{i j k}^{+} \Delta_{i j k}^{+}+\theta_{i j k}^{-} \Delta_{i j k}^{-}\right) \\
& -\sum_{j=1}^{J} \bar{f}_{i j}\left(x_{i j}^{\mathrm{New}}\right)-\sum_{j=1}^{J} \sum_{a \in L_{i}^{f}} \widehat{c}_{j a}\left(u_{j}^{f}\right) \\
& -\sum_{j=1}^{J} \sum_{k=1}^{n_{R}} \rho_{j}^{\mathrm{Re}} E\left(\widehat{r}_{k j i}\right)-\sum_{j=1}^{J} E\left(\bar{f}_{i j}^{\mathrm{Re}}\left(x_{i j}^{\mathrm{Re}}\right)\right) \\
& -\sum_{j=1}^{J} \sum_{k=1}^{n_{R}} \widehat{c}_{k j i}^{\mathrm{Re}} E\left(\widehat{r}_{k j i}\right)-\bar{\rho} \sum_{j=1}^{J} \sum_{k=1}^{n_{R}} E\left(\sum_{p^{f} \in P_{k}^{i}} x_{j p^{f}}-\widehat{r}_{k j i}\right),
\end{aligned}
$$

where (7), (9), and (14) hold for all decision variables defined by (4). By virtue of (12), (15)-(20), and (22), it is clear that $U_{i}$ is a function of the strategies of all firms in the entire CLSC network. That is,

$$
U_{i}=U_{i}(X)
$$

Now, in order to define the Cournot-Nash equilibrium of the CLSC network, we need to consider the following constraints involving firm $i(i=1, \ldots, I)$ :

$$
\begin{gathered}
x_{i j}^{\mathrm{New}} \leq \bar{x}, \\
\sum_{k=1}^{n_{R}} \sum_{p^{f} \in P_{k}^{i}} x_{j p^{f}} \leq x_{i j}^{\mathrm{New}}+E\left(x_{i j}^{\mathrm{Re}}\right), \\
E\left(\widehat{r}_{k j i}\right) \leq \sum_{p^{f} \in P_{k}^{i}} x_{j p^{f}},
\end{gathered}
$$

$$
x_{j p^{f}} \geq 0 \quad\left(\forall p^{f} \in \bigcup_{i=1}^{I} P^{i}\right), \quad x_{i j}^{\mathrm{New}} \geq 0 \text {. }
$$

Constraints (27), (28), and (29) are directly obtained from (5), (6), and (8), respectively; constraint (30) is due to the nonnegativity requirement imposed on all the decision variables.

There are $I$ oligopolistic firms in the market to form the CLSC network with perfect information shared by all the firms throughout the network. So the game is based on the oligopolistic Cournot pricing in a Cournot-Nash framework. Each firm in the CLSC network competes noncooperatively to maximize its own expected profit and selects its strategy vector till an equilibrium is established.

Now, we can define the Cournot-Nash equilibrium of the CLSC network according to Definitions 1, 2, and 3 given below. 
Definition 1 (a feasible strategy vector). For each $i(i=1$, $\ldots, I)$, a strategy vector $X_{i} \in K_{i}$ is said to be a feasible strategy vector, if $X_{i}$ satisfies constraints (27)-(30).

Definition 2 (the set of all feasible strategy patterns). Let $S$ be the set of all feasible strategy patterns defined by

$$
\begin{aligned}
& S \equiv\left\{\left(X_{1}, X_{2}, \ldots, X_{I}\right) \mid X_{i} \in K_{i}\right. \\
& \text { and constraints }(27)-(30) \text { holds }\}
\end{aligned}
$$

Definition 3 (the Cournot-Nash equilibrium of the CLSC network). A feasible strategy pattern $X^{*} \in S$ constitutes a Cournot-Nash equilibrium of the CLSC network, if the following inequality holds for all $i$ and for all feasible strategy vectors $X_{i}$ :

$$
U_{i}\left(X_{i}^{*}, \widehat{X}_{i}^{*}\right) \geq U_{i}\left(X_{i}, \widehat{X}_{i}^{*}\right)
$$

where $\widehat{X}_{i}^{*} \equiv\left(X_{1}^{*}, \ldots, X_{i-1}^{*}, X_{i+1}^{*}, \ldots, X_{I}^{*}\right)$.

Thus, an equilibrium is established if no firm in the CLSC network can unilaterally increase its expected profit (without violating feasibility) by changing any of its strategy, given that the strategies of the other firms do not change.

\section{The Variational Inequality Formulation}

To guarantee the existence of the Cournot-Nash equilibrium of the CLSC network, the following additional assumption is held.

Assumption B. (B1) The total operational cost $\widehat{c}_{j a}$ (a $\in L_{f}$, $j=1, \ldots, J)$ defined by (15) is a convex and continuously differentiable function of $u_{j}^{f}$, the vector consisting of all the forward link flows of product $j$.

(B2) The production cost $\bar{f}_{i j}(i=1, \ldots, I, j=1, \ldots, J)$ is a convex and continuously differentiable function of $x_{i j}^{\mathrm{New}}$, the new product $j$ manufactured by firm $i$.

Lemma 4. Suppose that Assumption A is satisfied, then the set of all feasible strategy patterns defined by Definition 2 is both a compact and convex subset of $K$.

Proof. The fact that $S$ is bounded follows easily from (30), (27), and (28). The fact that $S$ is closed and convex follows easily from (27), (28), (29), and (30). Hence, $S$ is both a compact and convex subset of $K$.

Lemma 5. Suppose that Assumption $A$ and Assumption $B$ are satisfied, then the expected profit function $U_{i}(\cdot)(i=1, \ldots, I)$ defined by (25) is continuously differentiable with respect to the decision variable $X$ defined by (4).

Proof. Firstly, from (9), (12), (13), (B1), and (14), it is clear that the first, second, and fourth terms of $U_{i}(\cdot)$ defined by (25) are continuous functions of $x_{j p^{f}}$, for each $j(j=1, \ldots, J)$ and $p^{f} \in P_{k}^{i}\left(k=1, \ldots, n_{R}\right)$.
By virtue of the fact that any two demands are independent of each other (from (A1)), the first term of $U_{i}(\cdot)$ can be expressed as

$$
\begin{aligned}
& E\left(\rho_{i j k}\left(\widehat{d}_{j}\right) \min \left\{v_{i j k}, \widehat{d}_{i j k}\right\}\right) \\
& =\int_{0}^{v_{i j k}} \widehat{d}_{i j k} \bar{G}\left(\widehat{d}_{i j k}\right) \mathscr{F}_{i j k}\left(\widehat{d}_{i j k}\right) \mathrm{d} \widehat{d}_{i j k} \\
& \quad+\int_{v_{i j k}}^{+\infty} v_{i j k} \bar{G}\left(\widehat{d}_{i j k}\right) \mathscr{F}_{i j k}\left(\widehat{d}_{i j k}\right) \mathrm{d} \widehat{d}_{i j k},
\end{aligned}
$$

where

$$
\begin{aligned}
\bar{G}\left(\widehat{d}_{i j k}\right) & \\
=\int_{0}^{+\infty} \ldots \int_{0}^{+\infty} & \rho_{i j k}\left(\widehat{d}_{111}, \ldots, \widehat{d}_{i j(k-1)}, \widehat{d}_{i j k}, \widehat{d}_{i j(k+1)}, \ldots, \widehat{d}_{I j n_{R}}\right) \\
& \times \mathscr{F}_{111}\left(\widehat{d}_{111}\right) \ldots \mathscr{F}_{i j(k-1)} \\
& \times\left(\widehat{d}_{i j(k-1)}\right) \mathscr{F}_{i j(k+1)}\left(\widehat{d}_{i j(k+1)}\right) \\
& \ldots \mathscr{F}_{I j n_{R}}\left(\widehat{d}_{I j n_{R}}\right) \mathrm{d} \widehat{d}_{111} \ldots \mathrm{d} \widehat{d}_{i j(k-1)} \mathrm{d} \widehat{d}_{i j(k+1)} \\
& \cdots \mathrm{d} \widehat{d}_{I j n_{R}} .
\end{aligned}
$$

Thus, we obtain from (33) that

$$
\begin{aligned}
& \frac{\partial E\left(\rho_{i j k}\left(\widehat{d}_{j}\right) \min \left\{v_{i j k}, \widehat{d}_{i j k}\right\}\right)}{\partial v_{i j k}} \\
& =\int_{v_{i j k}}^{+\infty} \bar{G}\left(\widehat{d}_{i j k}\right) \mathscr{F}_{i j k}\left(\widehat{d}_{i j k}\right) \mathrm{d} \widehat{d}_{i j k} .
\end{aligned}
$$

But, from (9), it is clear that

$$
\frac{\partial v_{i j k}}{\partial x_{j p^{f}}}=1
$$

for each $j(j=1, \ldots, J)$ and $p^{f} \in P_{k}^{i}\left(k=1, \ldots, n_{R}\right)$. Thus, from (35) and (36), the first partial derivative of the first term of $U_{i}(\cdot)$ becomes

$$
\begin{aligned}
& \frac{\partial E\left(\rho_{i j k}\left(\widehat{d}_{j}\right) \min \left\{v_{i j k}, \widehat{d}_{i j k}\right\}\right)}{\partial x_{j p^{f}}} \\
& =\int_{\sum_{p^{f} \in P_{k}^{i}} x_{j p} f}^{+\infty} \bar{G}\left(\widehat{d}_{i j k}\right) \mathscr{F}_{i j k}\left(\widehat{d}_{i j k}\right) \mathrm{d} \widehat{d}_{i j k},
\end{aligned}
$$

for each $j(j=1, \ldots, J)$ and $p^{f} \in P_{k}^{i}\left(k=1, \ldots, n_{R}\right)$. 
From (12), we have

$$
\begin{aligned}
\frac{\partial E\left(\Delta_{i j k}^{+}\right)}{\partial v_{i j k}} & =\frac{\partial}{\partial v_{i j k}} \int_{0}^{v_{i j k}}\left(v_{i j k}-\widehat{d}_{i j k}\right) \mathscr{F}_{i j k}\left(\widehat{d}_{i j k}\right) \mathrm{d} \widehat{d}_{i j k} \\
& =\int_{0}^{v_{i j k}} \mathscr{F}_{i j k}\left(\widehat{d}_{i j k}\right) \mathrm{d} \widehat{d}_{i j k}, \\
\frac{\partial E\left(\Delta_{i j k}^{-}\right)}{\partial v_{i j k}} & =\frac{\partial}{\partial v_{i j k}} \int_{v_{i j k}}^{+\infty}\left(\widehat{d}_{i j k}-v_{i j k}\right) \mathscr{F}_{i j k}\left(\widehat{d}_{i j k}\right) \mathrm{d} \widehat{d}_{i j k} \\
& =-\int_{v_{i j k}}^{+\infty} \mathscr{F}_{i j k}\left(\widehat{d}_{i j k}\right) \mathrm{d} \widehat{d}_{i j k} .
\end{aligned}
$$

Thus, from (13), (36), and (38), the first partial derivative of the second term of $U_{i}(\cdot)$ becomes

$$
\begin{gathered}
\frac{\partial E\left(\theta_{i j k}^{+} \Delta_{i j k}^{+}+\theta_{i j k}^{-} \Delta_{i j k}^{-}\right)}{\partial x_{j p^{f}}} \\
=\theta_{i j k}^{+} \int_{0}^{\sum_{p^{f} \in P_{k}^{i}} x_{j p} f} \mathscr{F}_{i j k}\left(\widehat{d}_{i j k}\right) \mathrm{d} \widehat{d}_{i j k} \\
\quad-\theta_{i j k}^{-} \int_{\sum_{p} \epsilon_{k}^{i} x_{j p} f}^{+\infty} \mathscr{F}_{i j k}\left(\widehat{d}_{i j k}\right) \mathrm{d} \widehat{d}_{i j k},
\end{gathered}
$$

for each $j(j=1, \ldots, J)$ and $p^{f} \in P_{k}^{i}\left(k=1, \ldots, n_{R}\right)$.

From (37) and (39), it is clear that the first and second terms of $U_{i}(\cdot)$ are continuously differentiable with respect to $x_{j p^{f}}$, for each $j(j=1, \ldots, J)$ and $p^{f} \in P_{k}^{i}\left(k=1, \ldots, n_{R}\right)$.

From (B1) and (14), the fourth term of $U_{i}(\cdot)$ is continuously differentiable with respect to $x_{j p^{f}}$, for each $j(j=$ $1, \ldots, J)$ and $p^{f} \in P_{k}^{i}\left(k=1, \ldots, n_{R}\right)$.

The third, fifth, sixth, and seventh terms of $U_{i}(\cdot)$ are independent of $x_{j p^{f}}$ and the last term of $U_{i}(\cdot)$ is a linear function of $x_{j p^{f}}$. Thus, all the terms in (25) are continuously differentiable with respect to $x_{j p^{f}}$, for each $j(j=1, \ldots, J)$ and $p^{f} \in P_{k}^{i}\left(k=1, \ldots, n_{R}\right)$.

From (B2), it is clear that the third term of $U_{i}(\cdot)$ is continuously differentiable with respect to $x_{i j}^{\mathrm{New}}$ and all the other terms are independent of $x_{i j}^{\mathrm{New}}$. Thus, all the terms in (25) are also continuously differentiable with respect to $x_{i j}^{\mathrm{New}}$, for each $j(j=1, \ldots, J)$. The proof is complete.

Lemma 6. The expected profit function $U_{i}(\cdot)(i=1, \ldots, I)$ defined by (25) is concave with respect to the decision variable $X$ defined by (4).

Proof. In order to prove that the first and second terms of $U_{i}(\cdot)$ are concave with respect to the decision variable $X$ defined by (4), we calculate the second partial derivative of these terms with respect to the decision variable $x_{j p^{f}}\left(j=1, \ldots, J, p^{f} \in\right.$ $\left.P_{k}^{i}, k=1, \ldots, n_{R}\right)$ as follows.
We obtain from (35) that

$$
\frac{\partial^{2} E\left(\rho_{i j k}\left(\widehat{d}_{j}\right) \min \left\{v_{i j k}, \widehat{d}_{i j k}\right\}\right)}{\partial v_{i j k}^{2}}=-\bar{G}\left(v_{i j k}\right) \mathscr{F}_{i j k}\left(v_{i j k}\right) \text {. }
$$

Thus, from (36) and (40), for each $j(j=1, \ldots, J)$ and $p^{f} \in P_{k}^{i}$ $\left(k=1, \ldots, n_{R}\right)$, the second partial derivative of the first term of $U_{i}(\cdot)$ becomes

$$
\frac{\partial^{2} E\left(\rho_{i j k}\left(\widehat{d}_{j}\right) \min \left\{v_{i j k}, \widehat{d}_{i j k}\right\}\right)}{\partial x_{j p^{f}}^{2}}=-\bar{G}\left(v_{i j k}\right) \mathscr{F}_{i j k}\left(v_{i j k}\right) \leq 0 .
$$

From (38), we have

$$
\begin{aligned}
& \frac{\partial^{2} E\left(\Delta_{i j k}^{+}\right)}{\partial v_{i j k}^{2}}=\mathscr{F}_{i j k}\left(v_{i j k}\right), \\
& \frac{\partial^{2} E\left(\Delta_{i j k}^{-}\right)}{\partial v_{i j k}^{2}}=\mathscr{F}_{i j k}\left(v_{i j k}\right) .
\end{aligned}
$$

Thus, from (13), (36), and (42), for each $j(j=1, \ldots, J)$ and $p^{f} \in P_{k}^{i}\left(k=1, \ldots, n_{R}\right)$, the second partial derivative of the second term of $U_{i}(\cdot)$ becomes

$$
\frac{\partial^{2} E\left(\theta_{i j k}^{+} \Delta_{i j k}^{+}+\theta_{i j k}^{-} \Delta_{i j k}^{-}\right)}{\partial x_{j p^{f}}^{2}}=\left(\theta_{i j k}^{+}+\theta_{i j k}^{-}\right) \mathscr{F}_{i j k}\left(v_{i j k}\right) \geq 0 .
$$

By virtue of (14), (15), and (B1), it is clear that $\left(-\widehat{c}_{j a}\left(u_{j}^{f}\right)\right)$ is concave with respect to $x_{j p^{f}}$, for each $j(j=1, \ldots, J)$ and $p^{f} \in P_{k}^{i}\left(k=1, \ldots, n_{R}\right)$.

Now, by virtue of (41), (43), the fact that the third, fifth, sixth, and seventh terms of $U_{i}(\cdot)$ are independent of $x_{j p}$, the fact that the fourth term of $U_{i}(\cdot)$ is concave with respect to $x_{j p^{f}}$, and the fact that the last term of $U_{i}(\cdot)$ is a linear function of $x_{j p^{f}}$, we conclude that $U_{i}(\cdot)$ is concave with respect to $x_{j p^{f}}$, for each $j(j=1, \ldots, J)$ and $p^{f} \in P_{k}^{i}\left(k=1, \ldots, n_{R}\right)$.

From (B2), it is clear that $\left(-\bar{f}_{i j}\left(x_{i j}^{\mathrm{New}}\right)\right)$ is concave with respect to $x_{i j}^{\mathrm{New}}$. Since $\left(-\bar{f}_{i j}\left(x_{i j}^{\mathrm{New}}\right)\right)$ is the only term in (25) involving $x_{i j}^{\mathrm{New}}$, we conclude that $U_{i}(\cdot)$ is concave with respect to $x_{i j}^{\mathrm{New}}$, for each $j(j=1, \ldots, J)$.

Hence, we conclude that the expected profit function $U_{i}(\cdot)$ $(i=1, \ldots, I)$ defined by $(25)$ is concave with respect to the decision variable $X$ defined by (4). The proof is complete.

We now give the variational inequality formulations of the CLSC network Cournot-Nash equilibrium in the following theorem.

Theorem 7. $X^{*} \in S$ is a CLSC network Cournot-Nash equilibrium according to Definition 3 if and only if it satisfies the variational inequality:

$$
-\sum_{i=1}^{I}\left\langle\nabla_{X_{i}} U_{i}\left(X^{*}\right), X_{i}-X_{i}^{*}\right\rangle \geq 0, \quad \forall X \in S,
$$


where $\langle\cdot, \cdot\rangle$ denotes the inner product in the corresponding Euclidean space and $\nabla_{X_{i}} U_{i}(X)$ denotes the gradient of $U_{i}(X)$ with respect to $X_{i}$.

Proof. In view of the fact that the set of all feasible patterns $S$ is both a compact and convex subset of $K$ and $U_{i}(\cdot)$ is concave and continuously differentiable with respect to the decision variable $X$ defined by (4) (from Lemmas 4-6), the proof of this theorem follows easily from [25].

Lemma 8. Variational inequality (44) for our model is equivalent to

Find $X^{*} \in S, \quad$ such that $\left\langle F\left(X^{*}\right), X-X^{*}\right\rangle \geq 0, \quad \forall X \in S$,

where $X \in \mathbb{R}^{\left(\sum_{i=1}^{I} n_{p} i+I J\right)}, F(X)=\left(F_{1}(X), F_{2}(X)\right), F_{1}(X) \in$ $\mathbb{R}^{\left(\sum_{i=1}^{I} n_{p^{i}} J\right)}, F_{2}(X) \in \mathbb{R}^{I J}$ such that the component of $F_{1}(X)$ corresponding to the variable $x_{j p^{f}}\left(\forall p^{f} \in P_{k}^{i}, \forall i, \forall j, \forall k\right)$ is

$$
\begin{gathered}
\sum_{a \in L_{i}^{f}} \frac{\partial \widehat{c}_{j a}\left(u_{j}^{f}\right)}{\partial x_{j p^{f}}}+\theta_{i j k}^{+} \int_{0}^{\sum_{p} f_{P_{k}^{i}} x_{j p} f} \mathscr{F}_{i j k}\left(\widehat{d}_{i j k}\right) \mathrm{d} \widehat{d}_{i j k} \\
\quad-\theta_{i j k}^{-} \int_{\sum_{p} f_{\in p_{k}^{i}} x_{j p} f}^{+\infty} \mathscr{F}_{i j k}\left(\widehat{d}_{i j k}\right) \mathrm{d} \widehat{d}_{i j k} \\
+\bar{\rho}-\int_{\sum_{p^{f} \in P_{k}^{i}} x_{j p} f}^{+\infty} \bar{G}\left(\widehat{d}_{i j k}\right) \mathscr{F}_{i j k}\left(\widehat{d}_{i j k}\right) \mathrm{d} \widehat{d}_{i j k},
\end{gathered}
$$

where $\bar{G}\left(\widehat{d}_{i j k}\right)$ is as defined in (34), and the component of $F_{2}(X)$ corresponding to the variable $x_{i j}^{N e w}(\forall i, \forall j)$ is

$$
\frac{\partial \bar{f}_{i j}\left(x_{i j}^{N e w}\right)}{\partial x_{i j}^{N e w}} \text {. }
$$

Proof. Since the first, second, fourth, and last terms of (25) are the only terms involving $x_{j p^{f}}$, we obtain from (25), (37), and (39) that

$$
\begin{aligned}
\frac{\partial U_{i}}{\partial x_{j p^{f}}}= & \int_{\sum_{p} f \in P_{k}^{i}}^{+\infty} \bar{G}\left(\hat{d}_{i j k}\right) \mathscr{F}_{i j k}\left(\widehat{d}_{i j k}\right) \mathrm{d} \widehat{d}_{i j k} \\
& -\theta_{i j k}^{+} \int_{0}^{\sum_{p} f_{\epsilon P_{k}^{i}} x_{j p^{f}}} \mathscr{F}_{i j k}\left(\widehat{d}_{i j k}\right) \mathrm{d} \widehat{d}_{i j k} \\
& +\theta_{i j k}^{-} \int_{\sum_{p} f \in P_{k}^{i} x_{j p}^{f}}^{+\infty} \mathscr{F}_{i j k}\left(\widehat{d}_{i j k}\right) \mathrm{d} \widehat{d}_{i j k} \\
& -\sum_{a \in L_{i}^{f}} \frac{\partial \widehat{c}_{j a}\left(u_{j}^{f}\right)}{\partial x_{j p^{f}}}-\bar{\rho}
\end{aligned}
$$

for each $j(j=1, \ldots, J)$ and $p^{f} \in P_{k}^{i}(i=1, \ldots, I, k=$ $\left.1, \ldots, n_{R}\right)$.

Since the third term of (25) is the only term involving $x_{i j}^{\mathrm{New}}$, we have

$$
\frac{\partial U_{i}}{\partial x_{i j}^{\mathrm{New}}}=-\frac{\partial \bar{f}_{i j}\left(x_{i j}^{\mathrm{New}}\right)}{\partial x_{i j}^{\mathrm{New}}}
$$

for each $x_{i j}^{\mathrm{New}}(i=1, \ldots, I, j=1, \ldots, J)$.
By virtue of (44), (48), and (49), we obtain variational inequality (45). Thus, the proof is complete.

\section{Existence and Monotonicity Results}

In this section, we establish the existence and monotonicity results of variational inequality (45), the solution of which is a CLSC network Cournot-Nash equilibrium of our model.

From Lemma 4, the feasible set of variational inequality (45) is both a compact and convex subset of $K$. From Lemma 5 , the expected profit function $U_{i}(\cdot)(i=1, \ldots, I)$ defined by (25) is continuously differentiable with respect to the decision variable $X$ defined by (4). Thus, we have the following theorem.

Theorem 9 (existence). Variational inequality (45) admits at least one solution.

Proof. Since $S$ is compact and convex and $F(X)$ is continuous, Theorem 9 follows from [26, Theorem 3.1, page 12].

Theorem 10 (monotonicity). Suppose that Assumptions A and $B$ are satisfied, then the vector function $F(X)$ of the variational inequality (45) is monotone; that is,

$$
\left\langle F\left(X^{\prime}\right)-F\left(X^{\prime \prime}\right), X^{\prime}-X^{\prime \prime}\right\rangle \geq 0, \quad \forall X^{\prime}, X^{\prime \prime} \in S .
$$

Proof. Let $X^{\prime} \in S$ and $X^{\prime \prime} \in S$. Variational inequality (50) has the following deduction:

$$
\begin{aligned}
& \left\langle F\left(X^{\prime}\right)-F\left(X^{\prime \prime}\right), X^{\prime}-X^{\prime \prime}\right\rangle \\
& =\sum_{i=1}^{I} \sum_{j=1}^{J} \sum_{k=1}^{n_{R}} \sum_{p^{f} \in P_{k}^{i}}\left[\sum_{a \in L_{i}^{f}} \frac{\partial \widehat{c}_{j a}\left(u_{j}^{f^{\prime}}\right)}{\partial x_{j p^{f}}^{\prime}}-\sum_{a \in L_{i}^{f}} \frac{\partial \widehat{c}_{j a}\left(u_{j}^{f^{\prime \prime}}\right)}{\partial x_{j p^{f}}^{\prime \prime}}\right] \\
& \times\left[x_{j p^{f}}^{\prime}-x_{j p^{f}}^{\prime \prime}\right] \\
& +\sum_{i=1}^{I} \sum_{j=1}^{J} \sum_{k=1}^{n_{R}} \sum_{p^{f} \in P_{k}^{i}}\left[\theta_{i j k}^{+} \int_{0}^{\sum_{p} f_{\epsilon} p_{k}^{i} x_{j p}^{\prime} f} \mathscr{F}_{i j k}\left(\widehat{d}_{i j k}\right) \mathrm{d} \widehat{d}_{i j k}\right. \\
& \left.-\theta_{i j k}^{+} \int_{0}^{\sum_{p^{f} \in P_{k}^{j}} x_{j p^{f}}^{\prime \prime}} \mathscr{F}_{i j k}\left(\widehat{d}_{i j k}\right) \mathrm{d} \widehat{d}_{i j k}\right] \\
& \times\left[x_{j p^{f}}^{\prime}-x_{j p^{f}}^{\prime \prime}\right] \\
& +\sum_{i=1}^{I} \sum_{j=1}^{J} \sum_{k=1}^{n_{R}} \sum_{p^{f} \in P_{k}^{i}}\left[\theta_{i j k}^{-}\left(-\int_{\sum_{p} f \in P_{k}^{i} x_{j p}^{f}}^{+\infty} \mathscr{F}_{i j k}\left(\widehat{d}_{i j k}\right) \mathrm{d} \widehat{d}_{i j k}\right)\right. \\
& -\theta_{i j k}^{-}\left(-\int_{\sum_{p} f_{\in p_{k}^{i}} x_{j p}^{\prime \prime}{ }^{+\infty}} \mathscr{F}_{i j k}\right. \\
& \left.\left.\times\left(\widehat{d}_{i j k}\right) \mathrm{d} \widehat{d}_{i j k}\right)\right] \\
& \times\left[x_{j p^{f}}^{\prime}-x_{j p^{f}}^{\prime \prime}\right]
\end{aligned}
$$




$$
\begin{aligned}
& +\sum_{i=1}^{I} \sum_{j=1}^{J} \sum_{k=1}^{n_{R}} \sum_{p^{f} \in P_{k}^{i}}\left[-\int_{\sum_{p^{f} \in p_{k}^{i}} x_{j p}^{\prime}}^{+\infty} \bar{G}\left(\widehat{d}_{i j k}\right) \mathscr{F}_{i j k}\left(\widehat{d}_{i j k}\right) \mathrm{d} \widehat{d}_{i j k}\right.
\end{aligned}
$$



$$
\begin{aligned}
& \left.\times \mathscr{F}_{i j k}\left(\widehat{d}_{i j k}\right) \mathrm{d} \widehat{d}_{i j k}\right] \\
& \times\left[x_{j p^{f}}^{\prime}-x_{j p^{f}}^{\prime \prime}\right] \\
& +\sum_{i=1}^{I} \sum_{j=1}^{J}\left[\frac{\partial \bar{f}_{i j}\left(x_{i j}^{\mathrm{New}^{\prime}}\right)}{\partial x_{i j}^{\mathrm{New}}}-\frac{\partial \bar{f}_{i j}\left(x_{i j}^{\mathrm{New}^{\prime \prime}}\right)}{\partial x_{i j}^{\mathrm{New}^{\prime \prime}}}\right] \\
& \times\left[x_{i j}^{\mathrm{New}^{\prime}}-x_{i j}^{\mathrm{New}^{\prime \prime}}\right],
\end{aligned}
$$

where $\bar{G}\left(\widehat{d}_{i j k}\right)$ is as defined in (34).

By virtue of the fact that $\widehat{c}_{j a}\left(u_{j}^{f}\right)$ is a convex function (from (B1)), we obtain from (14) that

$$
\begin{aligned}
\sum_{i=1}^{I} \sum_{j=1}^{J} \sum_{k=1}^{n_{R}} \sum_{p^{f} \in P_{k}^{i}} & {\left[\sum_{a \in L_{i}^{f}} \frac{\partial \widehat{c}_{j a}\left(u_{j}^{f^{\prime}}\right)}{\partial x_{j p^{f}}^{\prime}}-\sum_{a \in L_{i}^{f}} \frac{\partial \widehat{c}_{j a}\left(u_{j}^{f^{\prime \prime}}\right)}{\partial x_{j p^{f}}^{\prime \prime}}\right] } \\
\times & {\left[x_{j p^{f}}^{\prime}-x_{j p^{f}}^{\prime \prime}\right] \geq 0 . }
\end{aligned}
$$

Since

$$
\begin{gathered}
\int_{0}^{\sum_{p} \epsilon_{k}^{i} x_{j p} f} \mathscr{F}_{i j k}\left(\widehat{d}_{i j k}\right) \mathrm{d} \widehat{d}_{i j k}=\operatorname{Pr}\left(\widehat{d}_{i j k} \leq \sum_{p^{f} \in P_{k}^{i}} x_{j p^{f}}\right), \\
-\int_{\sum_{p} f_{\epsilon P_{k}^{i}} x_{j p} f}^{+\infty} \mathscr{F}_{i j k}\left(\widehat{d}_{i j k}\right) \mathrm{d} \widehat{d}_{i j k}=\operatorname{Pr}\left(\widehat{d}_{i j k} \leq \sum_{p^{f} \in P_{k}^{i}} x_{j p^{f}}\right)-1,
\end{gathered}
$$

and the probability function $\operatorname{Pr}\left(\widehat{d}_{i j k} \leq \sum_{p^{f} \in P_{k}^{i}} x_{j p^{f}}\right)$ is an increasing function of $x_{j p^{f}}$, for each $j(j=1, \ldots, J), p^{f} \in P_{k}^{i}$ $\left(i=1, \ldots, I\right.$ and $\left.k=1, \ldots, n_{R}\right)$, we have

$$
\begin{aligned}
\sum_{i=1}^{I} \sum_{j=1}^{J} \sum_{k=1}^{n_{R}} \sum_{p^{f} \in P_{k}^{i}}\left[\theta_{i j k}^{+} \int_{0}^{\sum_{p} f_{\epsilon p_{k}^{i}} x_{j p f}^{\prime}} \mathscr{F}_{i j k}\left(\widehat{d}_{i j k}\right) \mathrm{d} \widehat{d}_{i j k}\right. \\
\left.-\theta_{i j k}^{+} \int_{0}^{\sum_{p^{f} \in p_{k}^{i}} x_{j p^{\prime}}^{\prime \prime}} \mathscr{F}_{i j k}\left(\widehat{d}_{i j k}\right) \mathrm{d} \widehat{d}_{i j k}\right] \\
\times\left[x_{j p^{f}}^{\prime}-x_{j p^{f}}^{\prime \prime}\right] \geq 0,
\end{aligned}
$$

$$
\begin{aligned}
& \sum_{i=1}^{I} \sum_{j=1}^{J} \sum_{k=1}^{n_{R}} \sum_{p^{f} \in P_{k}^{i}}\left[\theta_{i j k}^{-}\left(-\int_{\sum_{p} f_{\epsilon P_{k}^{i}} x_{j p}^{\prime} f}^{+\infty} \mathscr{F}_{i j k}\left(\widehat{d}_{i j k}\right) \mathrm{d} \widehat{d}_{i j k}\right)\right. \\
& \left.-\theta_{i j k}^{-}\left(-\int_{\sum_{p} f_{\in p_{k}} x_{j p}^{\prime \prime} f}^{+\infty} \mathscr{F}_{i j k}\left(\widehat{d}_{i j k}\right) \mathrm{d} \widehat{d}_{i j k}\right)\right] \\
& \times\left[x_{j p^{f}}^{\prime}-x_{j p^{f}}^{\prime \prime}\right] \geq 0 .
\end{aligned}
$$

From (37) and (41), we know that $\int_{\sum_{p^{f} \in p_{k}^{i}}^{+\infty} x_{j p} f} \bar{G}\left(\widehat{d}_{i j k}\right) \mathscr{F}_{i j k}$ $\left(\widehat{d}_{i j k}\right) \mathrm{d} \widehat{d}_{i j k}$ is a decreasing function of $x_{j p}$, for each $j(j=$ $1, \ldots, J)$ and $p^{f} \in P_{k}^{i}\left(i=1, \ldots, I, k=1, \ldots, n_{R}\right)$. Hence, we have

$$
\begin{aligned}
\sum_{i=1}^{I} \sum_{j=1}^{J} \sum_{k=1}^{n_{R}} \sum_{p^{f} \in P_{k}^{i}}\left[-\int_{\sum_{p} \in P_{k}^{i} x_{j p}^{\prime} f}^{+\infty} \bar{G}\left(\widehat{d}_{i j k}\right) \mathscr{F}_{i j k}\left(\widehat{d}_{i j k}\right) \mathrm{d} \widehat{d}_{i j k}\right. \\
\left.+\int_{\sum_{p} f_{\epsilon P_{k}^{i}} x_{j p f}^{\prime \prime}}^{+\infty} \bar{G}\left(\widehat{d}_{i j k}\right) \mathscr{F}_{i j k}\left(\widehat{d}_{i j k}\right) \mathrm{d} \widehat{d}_{i j k}\right] \\
\times\left[x_{j p^{f}}^{\prime}-x_{j p^{f}}^{\prime \prime}\right] \geq 0 .
\end{aligned}
$$

By virtue of the fact that $\bar{f}_{i j}\left(x_{i j}^{\mathrm{New}}\right)$ is a convex function (from (B2)), we have

$$
\begin{aligned}
\sum_{i=1}^{I} \sum_{j=1}^{J} & {\left[\frac{\partial \bar{f}_{i j}\left(x_{i j}^{\mathrm{New}^{\prime}}\right)}{\partial x_{i j}^{\mathrm{New}^{\prime}}}-\frac{\partial \bar{f}_{i j}\left(x_{i j}^{\mathrm{New}^{\prime \prime}}\right)}{\partial x_{i j}^{\mathrm{New}^{\prime \prime}}}\right] } \\
\times & {\left[x_{i j}^{\mathrm{New}^{\prime}}-x_{i j}^{\mathrm{New}^{\prime \prime}}\right] \geq 0 . }
\end{aligned}
$$

Thus, we conclude that (51) is nonnegative. The proof is complete.

Lemma 11. Suppose that Assumptions $A$ and $B$ are satisfied. Furthermore, suppose that all the total operational costs $\widehat{c}_{j a}$ $\left(a \in L^{f}, j=1, \ldots, J\right)$ and all the production costs $\bar{f}_{i j}(i=$ $1, \ldots, I, j=1, \ldots, J)$ are strictly convex functions, and then the vector function $F(X)$ of the variational inequality (45) is strictly monotone; that is, $\forall X^{\prime}, X^{\prime \prime} \in S$ with $X^{\prime} \neq X^{\prime \prime}$

$$
\left\langle F\left(X^{\prime}\right)-F\left(X^{\prime \prime}\right), X^{\prime}-X^{\prime \prime}\right\rangle>0 .
$$

Proof. Let the strategy vector of all firms $X$ be written in the form:

$$
X=\left(X_{1}, X_{2}\right),
$$

where

$$
\begin{aligned}
& X_{1}=\left\{\left(x_{j p^{f}}\right) \mid j=1, \ldots, J, p^{f} \in \bigcup_{i=1}^{I} P^{i}\right\} \in \mathbb{R}_{+}^{\sum_{i=1}^{I}\left(n_{p^{i}}\right) J}, \\
& X_{2}=\left\{\left(x_{i j}^{\mathrm{New}}\right) \mid i=1, \ldots, I, j=1, \ldots, J\right\} \in \mathbb{R}_{+}^{I J} .
\end{aligned}
$$

Let $X^{\prime}, X^{\prime \prime}$ be the given strategy vectors such that $X^{\prime} \neq X^{\prime \prime}$. Then $X_{1}^{\prime} \neq X_{1}^{\prime \prime}$ or $X_{2}^{\prime} \neq X_{2}^{\prime \prime}$. 
Case $1\left(X_{1}^{\prime} \neq X_{1}^{\prime \prime}\right)$. Since all the total operational costs $\widehat{c}_{j a}(a \in$ $\left.L^{f}, j=1, \ldots, J\right)$ are strictly convex functions, it follows from (14) that the first term of (51) is positive.

Case $2\left(X_{2}^{\prime} \neq X_{2}^{\prime \prime}\right)$. Since all the production costs $\bar{f}_{i j}(i=$ $1, \ldots, I, j=1, \ldots, J)$ are strictly convex functions, the last term of (51) is positive.

Thus, by using exactly the same argument as that given for the proof of Theorem 10, we obtain

$$
\left\langle F\left(X^{\prime}\right)-F\left(X^{\prime \prime}\right), X^{\prime}-X^{\prime \prime}\right\rangle>0 .
$$

The proof is complete.

Theorem 12 (uniqueness). Under the conditions of Lemma 11, there is a unique solution of variational inequality (45).

Proof. Theorem 12 follows from [26, Theorem 1.4, page 84].

\section{Computing the Cournot-Nash Equilibrium}

In this section, we use the logarithmic quadratic proximal (LQP) prediction-correction method [27] for solving the variational inequality (45) (the LQP prediction-correction method is an iterative method). For the sake of convenience of the readers, we first give a brief description of the LQP prediction-correction method.

Consider the following variational inequality problem:

Find $x^{*} \in S$ such that $\left\langle f\left(x^{*}\right), x-x^{*}\right\rangle \geq 0, \quad \forall x \in S$,

with linear constraints:

$$
S=\left\{x \in R^{n} \mid A x \leq b, x \geq 0\right\},
$$

where $f$ is a given continuously differentiable function in $\mathbb{R}^{n}$ and $A$ is an $m \times n$ matrix and $b \in \mathbb{R}^{m}$.

Then the above variational inequality problem can be transformed into the following variational inequality problem:

Find $u^{*} \in \Omega \quad$ such that $\left\langle F\left(u^{*}\right), u-u^{*}\right\rangle \geq 0, \quad \forall u \in \Omega$,

where

$$
u=\left(\begin{array}{l}
x \\
\lambda
\end{array}\right), \quad F(u)=\left(\begin{array}{c}
f(x)+A^{T} \lambda \\
-A x+b
\end{array}\right), \quad \Omega=\mathbb{R}_{+}^{n} \times \mathbb{R}_{+}^{m},
$$

and $\lambda$ is the Lagrange multiplier vector of the constraints $A x \leq b$.

Let $\mathbb{R}_{++}^{n}$ and $\mathbb{R}_{++}^{m}$ denote the interior of $\mathbb{R}_{+}^{n}$ and $\mathbb{R}_{+}^{m}$, respectively. For any given $x^{k} \in \mathbb{R}_{++}^{n}, \lambda^{k} \in \mathbb{R}_{++}^{m}, \beta^{k}>0$, the new iterate $\left(x^{k+1}, \lambda^{k+1}\right)$ can be obtained by solving the following system of equations:

$$
\beta^{k}\left(f(x)+A^{T} \lambda\right)+\left(x-x^{k}\right)+\mu\left(x^{k}-\widehat{X}_{k}^{2} x^{-1}\right)=0,
$$

$$
\lambda=P_{\mathbb{R}_{+}^{m}}\left[\lambda^{k}+\frac{\beta^{k}}{v}(A x-b)\right],
$$

where $0<\mu<1$ is a given fixed parameter, $v>0$ is a positive number, $\widehat{X}_{k}=\operatorname{diag}\left(x_{1}^{k}, x_{2}^{k}, \ldots, x_{n}^{k}\right), x^{-1}=\left(1 / x_{1}\right.$, $\left.1 / x_{2}, \ldots, 1 / x_{n}\right)$, and $P_{\mathbb{R}_{m}^{+}}[\lambda]$ denote the projection of $\lambda$ on $\mathbb{R}_{m}^{+}$; that is,

$$
P_{\mathbb{R}_{m}^{+}}[\lambda]=\arg \min \left\{\|Y-\lambda\| \mid Y \in \mathbb{R}_{m}^{+}\right\} .
$$

Here the term $\left(x-x^{k}\right)+\mu\left(x^{k}-\widehat{X}_{k}^{2} x^{-1}\right)$ is used to ensure that the new iterate $x^{k+1}$ is not too far from $x^{k}$. Thus the purpose of (65) is to keep $x^{k+1}$ not too far from $x^{k}$ and at the same time keep $f\left(x^{k+1}\right)+A^{T} \lambda^{k+1}$ close to zero, whereas the purpose of (66) is to keep both $\lambda^{k+1} \geq 0$ and $A x^{k+1}-b \geq 0$. However, system (65) and (66) cannot be easily solved simultaneously. To overcome this difficulty, in the prediction step of the LQP prediction-correction method, we first replace $x$ by the current iterate $x^{k}$ in (66) so that an approximation of $\lambda$, denoted by $\tilde{\lambda}^{k}$, can be easily obtained by solving

$$
\tilde{\lambda}^{k}=P_{\mathbb{R}_{m}^{+}}\left[\lambda^{k}+\frac{\beta^{k}}{\nu}\left(A x^{k}-b\right)\right] .
$$

Then we substitute $\lambda=\lambda^{k}$ into (65) to obtain an approximation of $x$, denoted by $\tilde{x}^{k}$. In other words, the prediction $\left(\tilde{x}^{k}, \tilde{\lambda}^{k}\right)$ can be obtained by solving the following system:

$$
\text { Prediction }\left\{\begin{array}{c}
\beta^{k}\left(f(x)+A^{T} \lambda\right)+\left(x-x^{k}\right) \\
-\mu\left(x^{k}-\widehat{X}_{k}^{2} x^{-1}\right) \approx 0, \\
\lambda=P_{\mathbb{R}_{+}^{m}}\left[\lambda^{k}+\frac{\beta^{k}}{\nu}\left(A x^{k}-b\right)\right] .
\end{array}\right.
$$

After we have obtained the prediction $\left(\tilde{x}^{k}, \tilde{\lambda}^{k}\right)$, the new iterate $\left(x^{k+1}, \lambda^{k+1}\right)$, called the corrector, can be obtained by solving the following system:

$$
\text { Correction }\left\{\begin{array}{c}
\alpha \beta^{k}\left(f\left(\tilde{x}^{k}\right)+A^{T} \widetilde{\lambda}^{k}\right)+\left(x-x^{k}\right) \\
-\mu\left(x^{k}-\widehat{X}_{k}^{2} x^{-1}\right)=0, \\
\lambda=P_{\mathbb{R}_{+}^{m}}\left[\lambda^{k}+\frac{\alpha}{v} \beta^{k}\left(A \widetilde{x}^{k}-b\right)\right],
\end{array}\right.
$$

where $\alpha$ is the step size.

From the above discussion, it is obvious that there are two main advantages for choosing the LQP predictioncorrection method for solving the variational inequality (45). From the computational point of view, each iteration of the LQP prediction-correction method consists of a prediction and a correction, both of which require very tiny computational load; thus the method is effectively applicable in practice. From the mathematical point of view, there are only two conditions to guarantee the convergence of the LQP prediction-correction method; namely, the function $F(X)$ in the variational inequality $(45)$ is continuous and monotone and the solution set of the variational inequality (45) is 
nonempty. Since the existence and the monotonicity of the variational inequality (45) for our CLSC model have been proved in Theorems 9 and 10, respectively, we can easily use the LQP prediction-correction method for finding the Cournot-Nash equilibrium defined by Definition 3, which is equivalent to the solution of the variational inequality (45).

For the sake of using the above LQP prediction-correction method for solving the variational inequality (45), we need to reformulate the set of all feasible strategy patterns defined by Definition 2 as follows:

$$
S=\left\{X \in \mathbb{R}^{\left(\sum_{i=1}^{I} n_{p^{i}} J+I J\right)} \mid A X \leq b, X \geq 0\right\},
$$

where $A \in \mathbb{R}^{\left(2+n_{R}\right) I J \times\left(\sum_{i=1}^{I} n_{p^{i}} J+I J\right)}, b \in \mathbb{R}^{\left(2+n_{R}\right) I J}$ are obtained from constraints (27)-(29).

Let

$e(X, \lambda) \equiv\left(\begin{array}{c}X-P_{\left(\sum_{i=1}^{I}{ }^{n} p^{i J+I J)}\right.}\left\{X-\left[F(X)+A^{T} \lambda\right]\right\} \\ \lambda^{-}-P_{\mathbb{R}_{+}^{\left(2+n_{R}\right) I J}}\{\lambda+[A X-b]\}\end{array}\right)$,

where the vector function $F(X)$ is as defined in (45), $\lambda \epsilon$ $\mathbb{R}_{+}^{\left(2+n_{R}\right) I J}$ is the Lagrange multiplier vector of the linear inequality constraints $A X-b \leq 0$, and $P_{K}$ denotes the projection on $K$. Choose $\|e(X, \lambda)\|<\varepsilon$ as the stopping criterion.

Now we are in a position to describe Algorithm 13, which is a modified version of the LQP prediction-correction method described above.

\section{Algorithm 13.}

Step 0. Let $\beta^{0}=1, \nu=1(>0), \eta=0.9(<1), \mu=0.1, \sigma=2.0(>$ 1) and $\varepsilon=10^{-8}$. Initialize $X^{0} \in \mathbb{R}^{\left(\sum_{i=1}^{I} n_{p^{i}} J+I J\right)}, \lambda^{0} \in \mathbb{R}_{+}^{\left(2+n_{R}\right) I J}$. Let $k=0$.

Step 1. If $\left\|e\left(X^{k}, \lambda^{k}\right)\right\|_{\infty} \leq \varepsilon$, then stop; else, go to Step 2 .

Step 2 (prediction step). Produce the predictor $\widetilde{X}^{k}, \widetilde{\lambda}^{k}$.

Step 2.1. Calculate $\widetilde{\lambda}^{k}$ and $\widetilde{X}^{k}$.

$$
\begin{gathered}
\tilde{\lambda}^{k}=P_{\mathbb{R}^{\left(2+n_{R}\right) I J}}\left[\lambda^{k}+\frac{\beta^{k}}{\nu}\left(A X^{k}-b\right)\right], \\
\widehat{s} \equiv(1-\mu) X^{k}-\beta^{k}\left(F\left(X^{k}\right)+A^{T} \widetilde{\lambda}^{k}\right), \\
\widetilde{X}_{l}^{k}=\frac{\left(\widehat{s}_{l}+\sqrt{\left(\widehat{s}_{l}\right)^{2}+4 \mu\left(X_{l}^{k}\right)^{2}}\right)}{2}, \quad l=1, \ldots, \sum_{i=1}^{I} n_{p^{i}} J+I J .
\end{gathered}
$$

Step 2.2. Update $\beta^{k}$.

Let

$$
r^{k}=\sqrt{\frac{v\left\|\xi_{1}^{k}\right\|^{2}+(1+\mu)\left\|\xi_{2}^{k}\right\|^{2}}{\nu\left(1-\mu^{2}\right)\left\|\pi_{1}^{k}\right\|^{2}+\nu^{2}(1-\mu)\left\|\pi_{2}^{k}\right\|^{2}}},
$$

where $\xi_{1}^{k}=\beta^{k}\left(F\left(X^{k}\right)-F\left(\widetilde{X}^{k}\right)\right) \in \mathbb{R}^{\sum_{i=1}^{I} n_{p^{i}} J+I J}, \xi_{2}^{k}=\beta^{k} A\left(X^{k}-\right.$ $\left.\widetilde{X}^{k}\right) \in \mathbb{R}^{\left(2+n_{R}\right) I J}$,

$$
\begin{aligned}
& \pi_{1}^{k}=X^{k}-\widetilde{X}^{k} \in \mathbb{R}^{\sum_{i=1}^{I} n_{p^{i}} J+I J}, \\
& \pi_{2}^{k}=\lambda^{k}-\widetilde{\lambda}^{k} \in \mathbb{R}^{\left(2+n_{R}\right) I J} .
\end{aligned}
$$

If $r^{k}>\eta$, then $\beta^{k}=\beta^{k} \times 0.8 / r^{k}$; go back to Step 2.1; else, go to Step 3.
Step 3. Adjust $\beta^{k}$ and $v$ as follows:

$$
\begin{aligned}
& \beta^{k+1}= \begin{cases}\frac{\beta^{k} \times 0.7}{r^{k}}, & \text { if } r^{k} \leq 0.5, \\
\beta^{k}, & \text { otherwise, }\end{cases} \\
& \nu= \begin{cases}0.5 v, & \text { if } \frac{\left\|\xi_{1}^{k}\right\|}{\sqrt{1+\mu}}>\frac{4\left\|\xi_{2}^{k}\right\|}{\sqrt{v}}, \\
2 v, & \text { if } \frac{4\left\|\xi_{1}^{k}\right\|}{\sqrt{1+\mu}}<\frac{\left\|\xi_{2}^{k}\right\|}{\sqrt{v}}, \\
\nu, & \text { otherwise. }\end{cases}
\end{aligned}
$$

Step 4. Calculate the step-size $\alpha^{k}$ in the correction step.

$$
\alpha^{k}=\frac{\gamma \alpha^{k *} \beta^{k}(1-\mu)}{(1+\mu)}
$$

where

$$
\begin{aligned}
\alpha^{k *}= & \left(\left(\pi_{1}^{k}+\xi_{1}^{k}\right)^{T} \pi_{1}^{k}+\left(\nu \pi_{2}^{k}+\xi_{2}^{k}\right)^{T} \pi_{2}^{k}\right) \\
& \times\left(\left[(1+\mu) \pi_{1}^{k}+\xi_{1}^{k}\right]^{T}\left[\pi_{1}^{k}+(1+\mu)^{-1} \xi_{1}^{k}\right]\right. \\
& \left.+\left(\nu \pi_{2}^{k}+\xi_{2}^{k}\right)^{T}\left(\pi_{2}^{k}+\nu^{-1} \xi_{2}^{k}\right)\right)^{-1} .
\end{aligned}
$$

Step 5 (correction step). Calculate the new iterate $X^{k+1}, \lambda^{k+1}$.

$$
\begin{gathered}
\widehat{s} \equiv(1-\mu) X^{k}-\alpha^{k}\left(F\left(\widetilde{X}^{k}\right)+A^{T} \widetilde{\lambda}^{k}\right), \\
X_{l}^{k+1}=\frac{\left(\widehat{s}_{l}+\sqrt{\left(\widehat{s}_{l}\right)^{2}+4 \mu\left(X_{l}^{k}\right)^{2}}\right)}{2}, \quad l=1, \ldots, \sum_{i=1}^{I} n_{p^{i}} J+I J, \\
\lambda^{k+1}=P_{\mathbb{R}_{+}^{\left(2+n_{R}\right) I I}}\left[\lambda^{k}+\frac{\alpha^{k}}{v}\left(A \widetilde{X}^{k}-b\right)\right] .
\end{gathered}
$$

Let $k=k+1$; go to Step 1 .

The convergence result for Algorithm 13 is stated in the following theorem.

Theorem 14 (convergence). Suppose that Assumptions $A$ and $B$ are satisfied, and then the sequence $\left\{X^{k}\right\}$ generated by Algorithm 13 converges to some $X^{*}$, where $X^{*}$ is the solution of the variational inequality (45).

Proof. The proof follows easily from Theorems 9 and 10 and Theorem 4.2 of [27].

\section{Numerical Examples}

In this section, numerical examples are solved to illustrate the efficiency of Algorithm 13 for finding the Cournot-Nash equilibrium of the CLSC network.

We consider a CLSC network involving oligopolistic competition among four firms. Each firm manufactures two products and has two manufacturers and two distribution 
centers to supply goods to three demand markets. Each firm also has two recovery centers for recycling the used products.

For each of the examples solved in this section, we use the same demand price functions, which are modified from [18].

For product 1, the corresponding demand price functions associated with firm $i(i=1,2,3,4)$ and demand market $R_{k}$ $(k=1,2,3)$ are as follows:

Firm 1: $\rho_{1 j 1}=-1.1 \widehat{d}_{1 j 1}-0.9 \widehat{d}_{2 j 1}-0.9 \widehat{d}_{3 j 1}-0.9 \widehat{d}_{4 j 1}+500$,

$$
\rho_{1 j 2}=-0.9 \widehat{d}_{1 j 2}-0.8 \widehat{d}_{2 j 2}-0.8 \widehat{d}_{3 j 2}-0.8 \widehat{d}_{4 j 2}+500,
$$$$
\rho_{1 j 3}=-1.0 \widehat{d}_{1 j 3}-0.9 \widehat{d}_{2 j 3}-0.9 \widehat{d}_{3 j 3}-0.9 \widehat{d}_{4 j 3}+500 ;
$$

Firm 2: $\rho_{2 j 1}=-0.7 \widehat{d}_{1 j 1}-1.1 \widehat{d}_{2 j 1}-0.7 \widehat{d}_{3 j 1}-0.7 \widehat{d}_{4 j 1}+490$,

$$
\rho_{2 j 2}=-0.7 \widehat{d}_{1 j 2}-0.9 \widehat{d}_{2 j 2}-0.7 \widehat{d}_{3 j 2}-0.7 \widehat{d}_{4 j 2}+490,
$$$$
\rho_{2 j 3}=-0.8 \widehat{d}_{1 j 3}-1.2 \widehat{d}_{2 j 3}-0.8 \widehat{d}_{3 j 3}-0.8 \widehat{d}_{4 j 3}+490 ;
$$

Firm 3: $\rho_{3 j 1}=-0.8 \widehat{d}_{1 j 1}-0.8 \widehat{d}_{2 j 1}-1.1 \widehat{d}_{3 j 1}-0.8 \widehat{d}_{4 j 1}+480$,

$$
\begin{aligned}
& \rho_{3 j 2}=-0.8 \widehat{d}_{1 j 2}-0.8 \widehat{d}_{2 j 2}-0.9 \widehat{d}_{3 j 2}-0.8 \widehat{d}_{4 j 2}+480, \\
& \rho_{3 j 3}=-0.9 \widehat{d}_{1 j 3}-0.9 \widehat{d}_{2 j 3}-1.2 \widehat{d}_{3 j 3}-0.9 \widehat{d}_{4 j 3}+480 ;
\end{aligned}
$$

Firm 4: $\rho_{4 j 1}=-0.7 \widehat{d}_{1 j 1}-0.7 \widehat{d}_{2 j 1}-0.7 \widehat{d}_{3 j 1}-1.0 \widehat{d}_{4 j 1}+470$,

$$
\begin{aligned}
& \rho_{4 j 2}=-0.7 \widehat{d}_{1 j 2}-0.7 \widehat{d}_{2 j 2}-0.7 \widehat{d}_{3 j 2}-0.9 \widehat{d}_{4 j 2}+470, \\
& \rho_{4 j 3}=-0.7 \widehat{d}_{1 j 3}-0.7 \widehat{d}_{2 j 3}-0.7 \widehat{d}_{3 j 3}-1.1 \widehat{d}_{4 j 3}+470 .
\end{aligned}
$$

For product 2, the corresponding demand price functions associated with firm $i(i=1,2,3,4)$ and demand market $R_{k}$ $(k=1,2,3)$ are as follows:

Firm 1: $\rho_{1 j 1}=-1.1 \widehat{d}_{1 j 1}-0.9 \widehat{d}_{2 j 1}-0.9 \widehat{d}_{3 j 1}-0.9 \widehat{d}_{4 j 1}+420$,

$$
\rho_{1 j 2}=-0.9 \widehat{d}_{1 j 2}-0.8 \widehat{d}_{2 j 2}-0.8 \widehat{d}_{3 j 2}-0.8 \widehat{d}_{4 j 2}+420,
$$$$
\rho_{1 j 3}=-1.0 \widehat{d}_{1 j 3}-0.9 \widehat{d}_{2 j 3}-0.9 \widehat{d}_{3 j 3}-0.9 \widehat{d}_{4 j 3}+420 ;
$$

Firm 2: $\rho_{2 j 1}=-0.7 \widehat{d}_{1 j 1}-1.1 \widehat{d}_{2 j 1}-0.7 \widehat{d}_{3 j 1}-0.7 \widehat{d}_{4 j 1}+430$,

$$
\begin{aligned}
& \rho_{2 j 2}=-0.7 \widehat{d}_{1 j 2}-0.9 \widehat{d}_{2 j 2}-0.7 \widehat{d}_{3 j 2}-0.7 \widehat{d}_{4 j 2}+430, \\
& \rho_{2 j 3}=-0.8 \widehat{d}_{1 j 3}-1.2 \widehat{d}_{2 j 3}-0.8 \widehat{d}_{3 j 3}-0.8 \widehat{d}_{4 j 3}+430 ;
\end{aligned}
$$

Firm 3: $\rho_{3 j 1}=-0.8 \widehat{d}_{1 j 1}-0.8 \widehat{d}_{2 j 1}-1.1 \widehat{d}_{3 j 1}-0.8 \widehat{d}_{4 j 1}+440$,

$$
\rho_{3 j 2}=-0.8 \widehat{d}_{1 j 2}-0.8 \widehat{d}_{2 j 2}-0.9 \widehat{d}_{3 j 2}-0.8 \widehat{d}_{4 j 2}+440,
$$$$
\rho_{3 j 3}=-0.9 \widehat{d}_{1 j 3}-0.9 \widehat{d}_{2 j 3}-1.2 \widehat{d}_{3 j 3}-0.9 \widehat{d}_{4 j 3}+440 ;
$$

Firm $4: \rho_{4 j 1}=-0.7 \widehat{d}_{1 j 1}-0.7 \widehat{d}_{2 j 1}-0.7 \widehat{d}_{3 j 1}-1.0 \widehat{d}_{4 j 1}+450$,

$$
\begin{aligned}
& \rho_{4 j 2}=-0.7 \widehat{d}_{1 j 2}-0.7 \widehat{d}_{2 j 2}-0.7 \widehat{d}_{3 j 2}-0.9 \widehat{d}_{4 j 2}+450, \\
& \rho_{4 j 3}=-0.7 \widehat{d}_{1 j 3}-0.7 \widehat{d}_{2 j 3}-0.7 \widehat{d}_{3 j 3}-1.1 \widehat{d}_{4 j 3}+450 .
\end{aligned}
$$

Assume that $\widehat{d}_{i j k}$ is uniformly distributed in $\left[0, \tau_{i j k}\right]$ with probability density function given by

$$
\mathscr{F}_{i j k}(x)= \begin{cases}\frac{1}{\tau_{i j k}}, & \text { if } x \in\left[0, \tau_{i j k}\right], \\ 0, & \text { if } x \in\left(\tau_{i j k},+\infty\right),\end{cases}
$$

where for product $j=1, \tau_{1 j k}=28, \tau_{2 j k}=27, \tau_{3 j k}=26$, $\tau_{4 j k}=25(k=1,2,3)$ and for product $j=2, \tau_{1 j k}=20$, $\tau_{2 j k}=19, \tau_{3 j k}=18, \tau_{4 j k}=17(k=1,2,3)$.

Assume that $\widehat{r}_{k j i}$ (the returned product $j(j=1,2)$ from demand market $R_{k}(k=1,2,3)$ to firm $\left.i(i=1,2,3,4)\right)$ is uniformly distributed in $[0,8]$.

Example 15. In this example, the demand price functions are as given in (80)-(81). The cost functions, which are modified from those given in [4], are as follows:

$$
\begin{gathered}
\widehat{c}_{j a}\left(u_{j}^{f}\right)=2\left(u_{j a}^{f}\right)^{2}+\left(u_{j a}^{f}\right), \quad \forall a \in L_{f}^{i} ; \\
\bar{f}_{i j}\left(x_{i j}^{\mathrm{New}}\right)=2.5\left(x_{i j}^{\mathrm{New}}\right)^{2}+2 x_{i j}^{\mathrm{New}}, \\
\bar{f}_{i j}^{\mathrm{Re}}\left(x_{i j}^{\mathrm{Re}}\right)=\left(x_{i j}^{\mathrm{Re}}\right)^{2}+0.5 x_{i j}^{\mathrm{Re}},
\end{gathered}
$$

where $i=1,2,3,4, j=1,2$, and $k=1,2,3$.

The values of the parameters are as follows: $\theta_{i j k}^{+}$(unit penalty incurred on firm $i$ due to excessive supply of product $j$ to demand market $R_{k}$ ) $=20, \theta_{i j k}^{-}$(unit penalty incurred on firm $i$ due to insufficient supply of product $j$ to demand market $R_{k}$ ) $=20, \widehat{c}_{k j i}^{\mathrm{Re}}$ (transportation cost per item of returned product $j$ from demand market $R_{k}$ to firm $i$ ) $=0.5, \rho_{j}^{\mathrm{Re}}$ (purchase cost per item of returned product $j$ from demand market $R_{k}$ to firm $i$ ) $=10$, and $\bar{\rho}$ (disposal fee per item of the used products at the landfill site) $=10$, where $i=1,2,3,4, j=1,2$, and $k=1,2,3$.

In Example 15, all the conditions of Theorem 12 are satisfied. We solve this example by Algorithm 13 (using Matlab) to obtain the Cournot-Nash equilibrium of the CLSC network. The Cournot-Nash equilibrium representing the optimal strategies of all the firms is presented in the fourth column of Table 1 . The expected profits of firm $i(i=1,2,3,4)$, denoted by $U_{i}^{*}$, at the Cournot-Nash equilibrium are

$$
\begin{array}{ll}
U_{1}^{*}=13093, & U_{2}^{*}=12988, \\
U_{3}^{*}=12536, & U_{4}^{*}=12483 .
\end{array}
$$

Here is a summary of the numerical results for Example 15.

For both Product 1 and Product 2, the situation of the optimal strategies at Cournot-Nash equilibrium is as follows.

Firm 1 should manufacture the largest amount of new products as well as provide the largest amount of forward product flows to each of the demand markets $R_{1}, R_{2}$, and $R_{3}$, followed by Firm 2, Firm 3, and Firm 4. Furthermore, Firm 1 should receive the largest amount of profit, followed by Firm 2, Firm 3, and Firm 4. 
TABle 1: Optimal strategies for all firms for Examples 15 and 16.

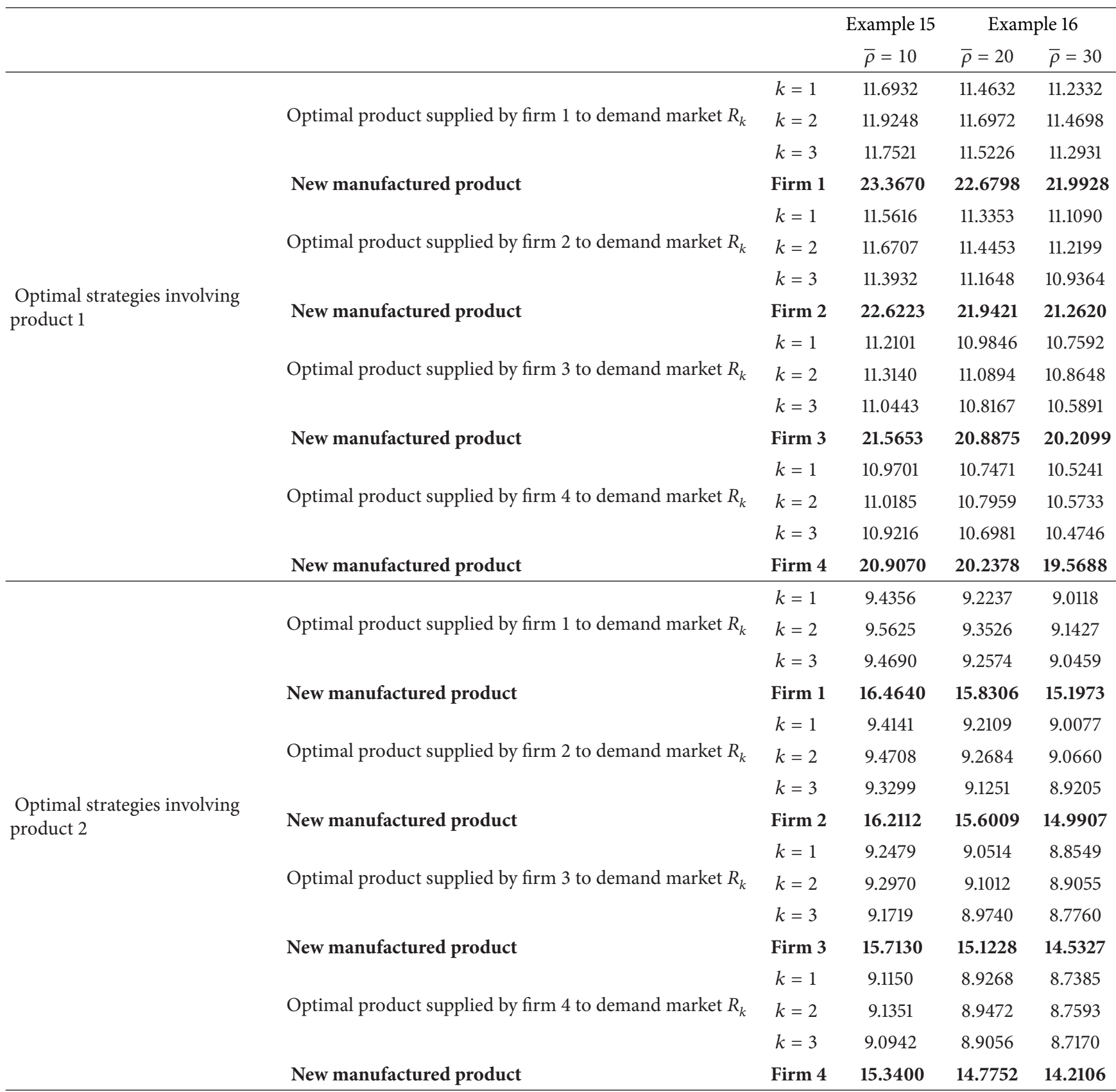

In the next example, we use the parameter $\bar{\rho}$ to perform sensitivity analysis. By fixing the other parameters, we study the effect of changing the parameter $\bar{\rho}$ on the Cournot-Nash equilibrium together with the optimal expected profits of all firms.

Example 16. Same as Example 15, except that $\bar{\rho}=10$ in Example 15 is first replaced by $\bar{\rho}=20$ and then by $\bar{\rho}=30$ in this example. (In other words, we now increase the disposal fee per item by $100 \%$ and $200 \%$ ). Thus, the main purpose of this example is to test the effect of increasing the disposal fee per item on the firms' optimal strategies and the firms' expected profits.
The Cournot-Nash equilibrium representing the optimal strategies of all the firms when $\bar{\rho}=20$ is presented in the fifth column of Table 1 , and the expected profits of firm $i$ ( $i=$ $1,2,3,4)$, denoted by $U_{i}^{*}$, at the Cournot-Nash equilibrium are

$$
\begin{array}{ll}
U_{1}^{*}=12701, & U_{2}^{*}=12606, \\
U_{3}^{*}=12170, & U_{4}^{*}=12127 .
\end{array}
$$

The Cournot-Nash equilibrium representing the optimal strategies of all the firms when $\bar{\rho}=30$ is presented in the sixth column of Table 1 , and the expected profits of 
firm $i(i=1,2,3,4)$, denoted by $U_{i}^{*}$, at the Cournot-Nash equilibrium are

$$
\begin{array}{ll}
U_{1}^{*}=12323, & U_{2}^{*}=12237, \\
U_{3}^{*}=11816, & U_{4}^{*}=11783 .
\end{array}
$$

From the fourth, fifth, and sixth columns of Table 1 and the value of the expected profits given above, we observe the following.

(i) When $\bar{\rho}$ is increased, both the optimal product flows and the new manufactured products of each firm decrease.

(ii) When $\bar{\rho}$ is increased, the expected profit of each firm also decreases.

Remark 17. From the above two examples, we conclude that the change in disposal fee per item of used products not only affects the firms' optimal strategies, but also their expected profits.

\section{Conclusion and Suggestions for Further Studies}

In this paper, we develop a multiproduct CLSC network equilibrium model with uncertain demands and product returns. This model belongs to the context of oligopolistic firms that compete noncooperatively in a Cournot-Nash framework under a stochastic environment. The CournotNash equilibrium conditions are solved by using the LQP prediction-correction method. Finally, numerical examples are presented to test the efficiency of the algorithm for finding the Cournot-Nash equilibrium of the CLSC network. In the future, we would like to extend our method to solve a multiperiod CLSC network equilibrium model involving oligopolistic competition among firms.

\section{Conflict of Interests}

The authors declare that there is no conflict of interests regarding the publication of this paper.

\section{Acknowledgments}

This research was supported by the General Research Fund (Project no. Poly U 5405/11 H) of the Research Grants Council, Hong Kong, and the Research Committee of the Hong Kong Polytechnic University. It was also supported by the National Natural Science Foundation of China (no. 71371102) and the Natural Science Foundation of Shandong Province of China (ZR2013GQ007).

\section{References}

[1] A. Nagurney, J. Dong, and D. Zhang, "A supply chain network equilibrium model," Transportation Research Part E: Logistics and Transportation Review, vol. 38, no. 5, pp. 281-303, 2002.
[2] J. Dong, D. Zhang, and A. Nagurney, "A supply chain network equilibrium model with random demands," European Journal of Operational Research, vol. 156, no. 1, pp. 194-212, 2004.

[3] A. Nagurney and F. Toyasaki, "Reverse supply chain management and electronic waste recycling: a multitiered network equilibrium framework for E-cycling," Transportation Research Part E: Logistics and Transportation Review, vol. 41, no. 1, pp. $1-28,2005$.

[4] D. Hammond and P. Beullens, "Closed-loop supply chain network equilibrium under legislation," European Journal of Operational Research, vol. 183, no. 2, pp. 895-908, 2007.

[5] G.-F. Yang, Z.-P. Wang, and X.-Q. Li, "The optimization of the closed-loop supply chain network," Transportation Research Part E: Logistics and Transportation Review, vol. 45, no. 1, pp. 16-28, 2009.

[6] G. M. Korpelevich, "An extragradient method for finding saddle points and for other problems," Matecon, vol. 12, no. 4, pp. 747756, 1976.

[7] European Union, "Directive 2002/96/EC of the European Parliament and of the Council of 27 January 2003 on waste electrical and electronic equipment (WEEE)," Oficial Journal of the European Union, L 37/34.

[8] S. Gupta and O. D. Palsule-Desai, "Sustainable supply chain management: review and research opportunities," IIMB Management Review, vol. 23, no. 4, pp. 234-245, 2011.

[9] J.-B. Sheu and W. K. Talley, "Green supply chain management: trends, challenges, and solutions," Transportation Research Part E: Logistics and Transportation Review, vol. 47, no. 6, pp. 791792, 2011.

[10] S. Seuring, "A review of modeling approaches for sustainable supply chain management," Decision Support Systems, vol. 54, no. 4, pp. 1513-1520, 2013.

[11] A. A. Cournot, Researches into the Mathematical Principles of the Theory of Wealth, English Translation, MacMillan, London, $\mathrm{UK}, 1838$.

[12] A. Barbagallo and M.-G. Cojocaru, "Dynamic equilibrium formulation of the oligopolistic market problem," Mathematical and Computer Modelling, vol. 49, no. 5-6, pp. 966-976, 2009.

[13] A. Barbagallo and P. Mauro, "Time-dependent variational inequality for an oligopolistic market equilibrium problem with production and demand excesses," Abstract and Applied Analysis, vol. 2012, Article ID 651975, 35 pages, 2012.

[14] G. Brown, J. Keegan, B. Vigus, and K. Wood, "The Kellogg Company optimizes production, inventory, and distribution," Interfaces, vol. 31, no. 6, pp. 1-15, 2001.

[15] A. Nagurney, "Supply chain network design under profit maximization and oligopolistic competition," Transportation Research Part E: Logistics and Transportation Review, vol. 46, no. 3, pp. 281-294, 2010.

[16] J. F. Nash, "Equilibrium points in N-Person games," Proceedings of the National Academy of Sciences, vol. 36, pp. 48-49, 1950.

[17] J. F. Nash, "Noncooperative games," Annals of Mathematics, vol. 54, pp. 286-298, 1951.

[18] A. H. Masoumi, M. Yu, and A. Nagurney, "A supply chain generalized network oligopoly model for pharmaceuticals under brand differentiation and perishability," Transportation Research Part E: Logistics and Transportation Review, vol. 48, no. 4, pp. 762-780, 2012.

[19] A. Nagurney and M. Yu, "Sustainable fashion supply chain management under oligopolistic competition and brand differentiation," International Journal of Production Economics, vol. 135, no. 2, pp. 532-540, 2012. 
[20] M. Yu and A. Nagurney, "Competitive food supply chain networks with application to fresh produce," European Journal of Operational Research, vol. 224, pp. 273-282, 2013.

[21] P. Dupuis and A. Nagurney, "Dynamical systems and variational inequalities," Annals of Operations Research, vol. 44, no. 1, pp. 942, 1993.

[22] Q. Qiang, K. Ke, T. Anderson, and J. Dong, “The closed-loop supply chain network with competition, distribution channel investment, and uncertainties," Omega, vol. 41, pp. 186-194, 2013.

[23] J. M. Shi, G. Q. Zhang, and J. C. Sha, "Optimal production and pricing policy for a closed loop system," Resources, Conservation and Recycling, vol. 55, no. 6, pp. 639-647, 2011.

[24] J. M. Shi, G. Q. Zhang, and J. C. Sha, "Optimal production planning for a multi-product closed loop system with uncertain demand and return," Computers and Operations Research, vol. 38, no. 3, pp. 641-650, 2011

[25] D. Gabay and H. Moulin, "On the uniqueness and stability of Nash equilibria in noncooperative games," in Applied Stochastic Control of Econometrics and Management Science, A. Bensoussan, P. Kleindorfer, and C. S. Tapiero, Eds., pp. 271-294, NorthHolland, Amsterdam, The Netherlands, 1980.

[26] D. Kinderlehrer and G. Stampacchia, An Introduction to Variational Inequalities and Their Application, Academic Press, New York, NY, USA, 1980.

[27] B. S. He and X. M. Yuan, "A Logarithmic-Quadratic proximal prediction-correction method for structured monotone variational inequalities," Computational Optimization and Applications, vol. 35, no. 1, pp. 19-46, 2006. 


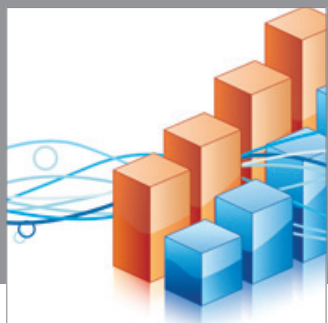

Advances in

Operations Research

mansans

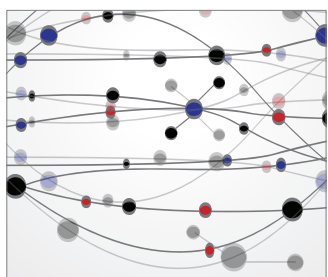

The Scientific World Journal
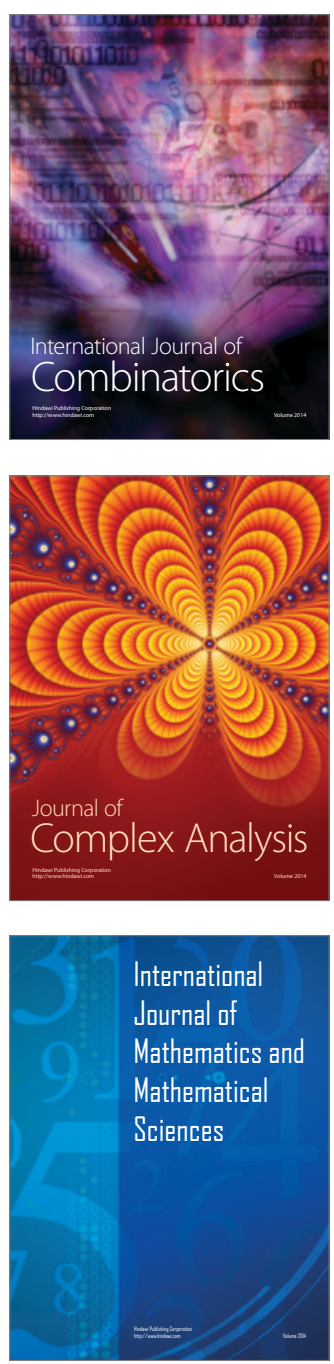
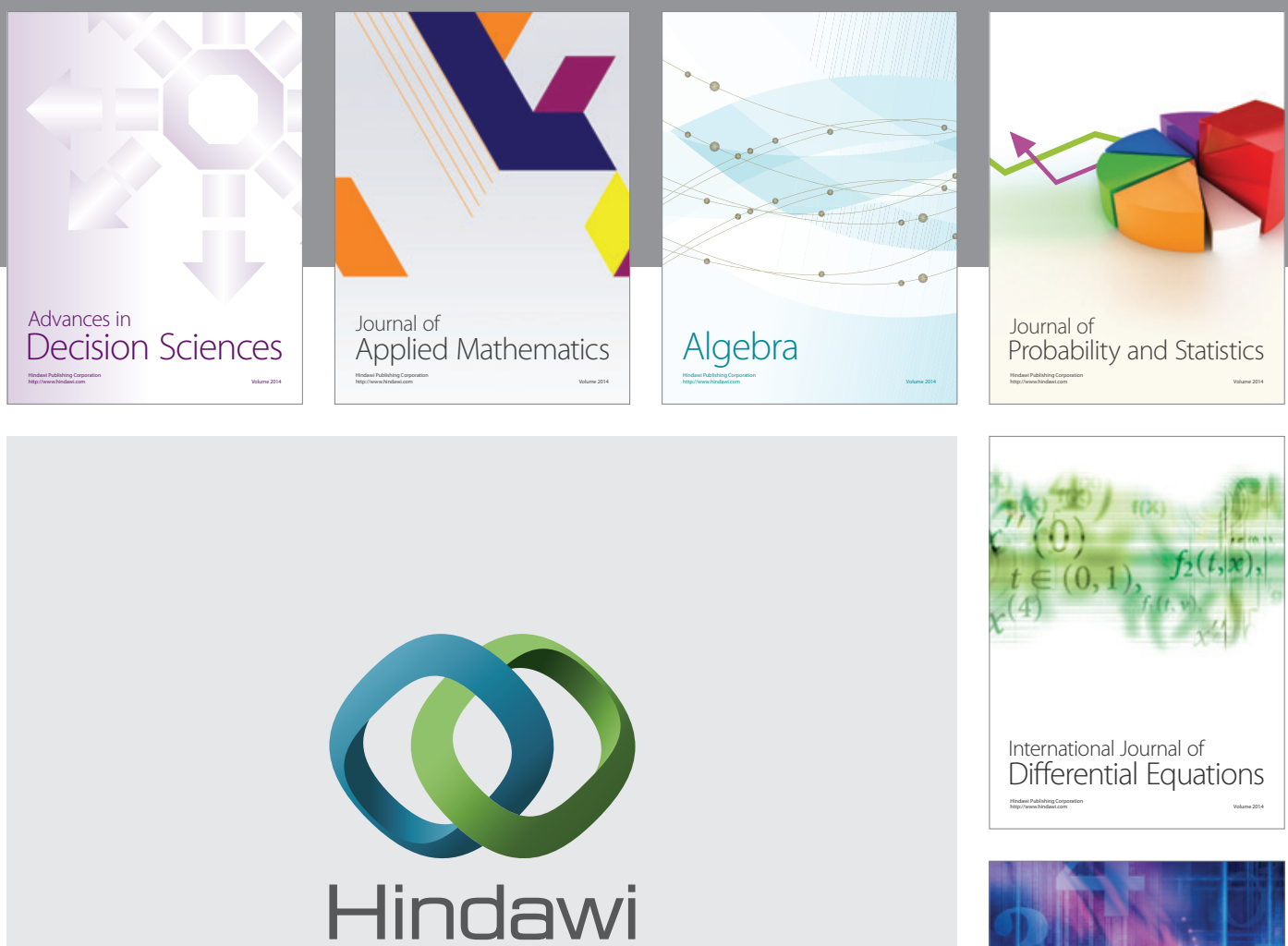

Submit your manuscripts at http://www.hindawi.com
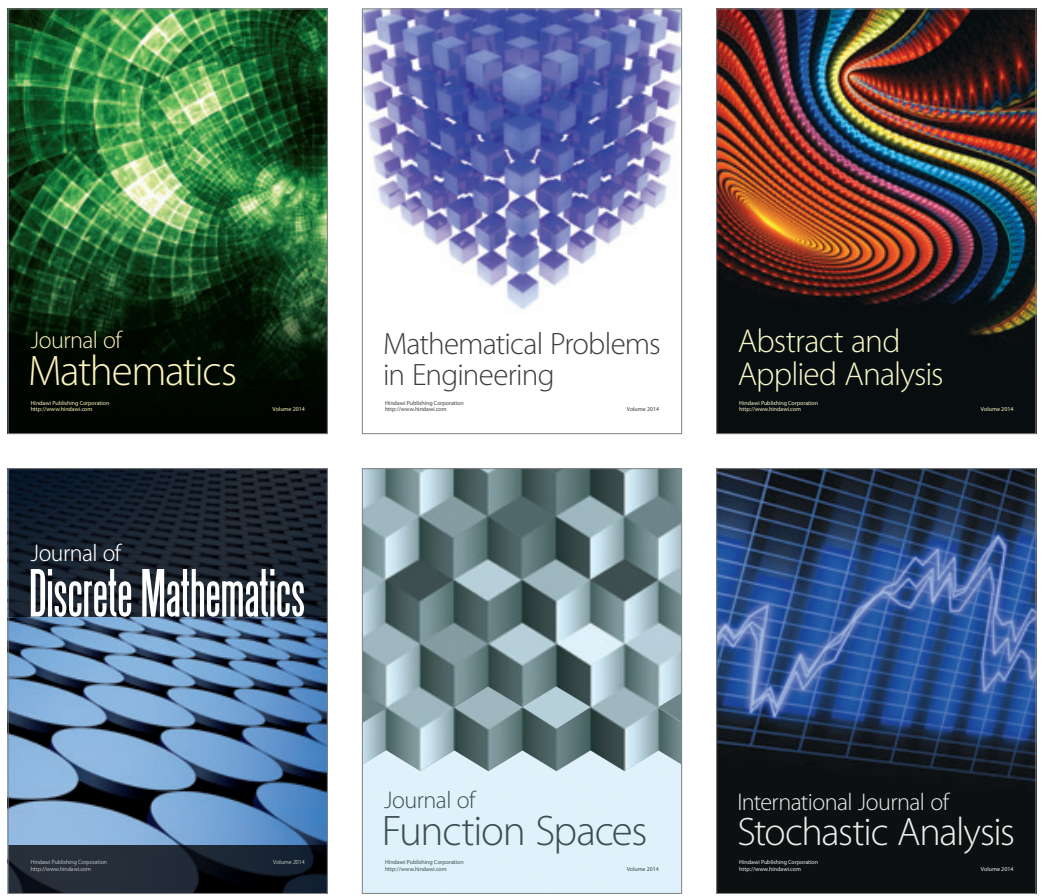

Journal of

Function Spaces

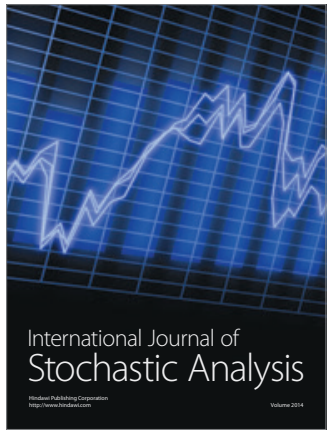

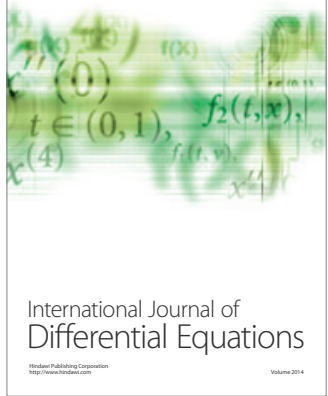
